الخصائص السيكومترية المختصرة المعربّة لمقياس الرضا عن الحياة المتعدد الأبعاد للطلبة (BMSLSS) فِ البيئة السورية

\author{
إمطانيوس ميخائيل \\ michaeel@scs-net.org \\ جامعة دمشق

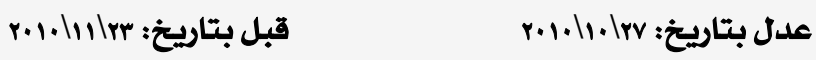

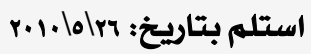

أعدت الصورة المختصرة لمقياس الرضا عن الحياة المتعدد الأبعاد للطلبة BMSLSS بهدف توفير أداة سريعة لتقدير

درجات الرضا عن الحياة لدى طلبة المرحلة قبل الجامعية ِِّ مجالات محددة هي: الأسـرة، والأصدقاء، والمدرسلة، وبيئة الحياة،

والذات. وقد هدفت هذه الدراسـة إلى إعداد صورة عربيـة موازيـة لتلك الصورة والتحقق من كفاءتها السيكومتريـة لدى طلبة

الثانوية والجامعة على حلد سواء، ومن أجل ذلك استخدمت طرائق متعددة ِِّ دراسلة الثبات والصدق لهذه الصورة، كما

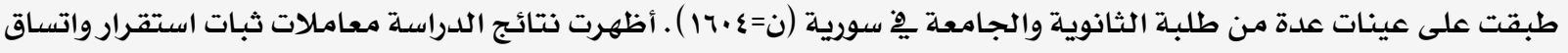

مناسبـة للصورة العربية المقترحة، كما وفرت دلالات لصدقها التقاريي والتباعدي باستخدام عدد من المقاييس المحكية،

ولصدق التكوين الفرضي عن طريق دراسة الارتباطات البينية (أو الداخلية) للهقاييس الفرعية الخمسة لهذه الصورة،

وارتباطات هذه المقاييس الفرعية ذاتها مـع نظرائها ِِ المقياس الكامل (أو الصورة الأم). هذا بالإضـافة إلى ارتباطاتها بالدرجات

التحصيلية مها يوفر دعماً إضافياً لصدقها. وتشير نتائج هذه الدراسـة إلى ملائمة الصورة العربية المختصرة للاستخدام،

وتتلخص المقترحات التي طرحها الباحث ِِِ إجراء المزيد من الدراسـات السيكومترية لها، وإلى إمكانية استخدامها ِِِ البحوث

المسحية وبحوث المقارنات الثقافية (أو الحضـاريـة).

الكلمات المفتاحية : مقياس الرضا عن الحياة، تقدير (أو قياس ) الرضا عن الحياة، قياس الشخصية، الطلبة السوريون.

\title{
A Study of The Brief Multidimensional Students' life Satisfaction Scale (BMSLSS) with Syrian Secondary and University Students \\ Emtanuos Michaeel \\ Damascus University
}

The Brief Multidimensional Students' Life Satisfaction Scale (BMSLSS) is a self-report measure developed to assess life satisfaction among students in five specific domains: family, friends, school, self, and daily life. The purpose of the current study was to develop an Arabic version of this measure and to investigate its psychometric properties with Syrian secondary students as well as university students. With a sample of $(\mathrm{N}=1604)$, several methods were used to estimate the reliability and validity of the measure. The results showed satisfactory test-retest reliability and internal consistency coefficients. Also, the results provided evidence for the convergent and divergent validity. Further evidence for the construct validity of the instrument was provided by studying the inter-correlations of its five subscales as well as the correlations of these subscales with the subscales of the entire instrument. At the same time, validity was supported by the correlations of the five subscales with achievement. In sum, the findings of this study show that the psychometric properties obtained from administering the instrument to a sample of secondary school and university students meets acceptable levels. Recommendations were made to conduct further psychometric studies upon the Arabic version of BMSLSS and to administer this version in cross- cultural studies.

Keywords: Life Satisfaction Scale, assessment of life satisfaction, Syrian university students, Syrian secondary school students. 
كالقلق، والاكتئاب، والتشاؤم، والانطواء الاجتماعي

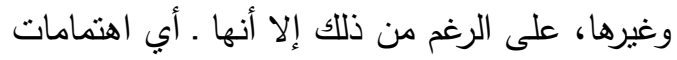

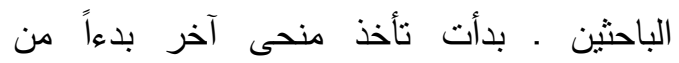
ثمانينيات القرن الماضي، وقد حدث ذلك بعد أن

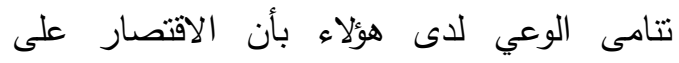
المظاهر السلبية والمرضية للشخصية بعد أن تكون

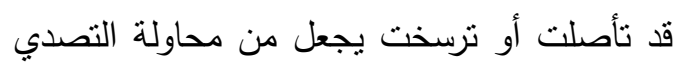

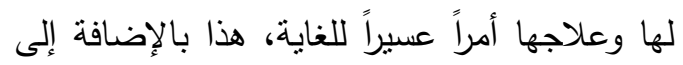
أن التشديد على دراسة المظاهر الإيجابية للشخصية قد يسفر عن الكثير من نقاط القوة التي يمكن توظيفها مباشرة في تخطي الصعوبات ومواجهة نقاط الضعف أو غيرها من المظاهر

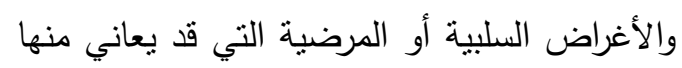
الفرد، هذا من جهة؛ ومن جهة أخرى فإن دراسة

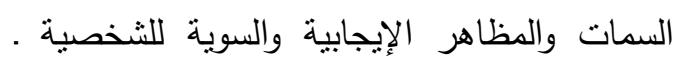

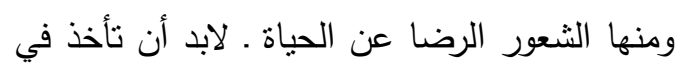

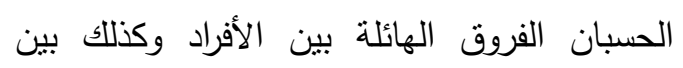
المجتمعات، إضافة إلى الفروق بين الجماعات

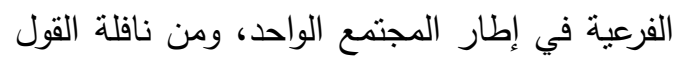

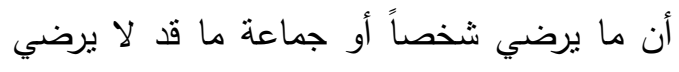

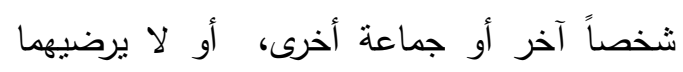
بالقدر نفسه. فقد ينظر البعض إلى الثروة المادية

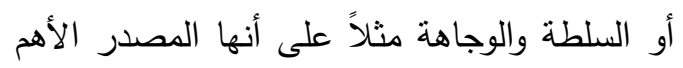
للرضا عن الحياة، في حين أن هنالك من يعطي الحرية الثخصبة أو الصحة واللياقة البدنية الرتبة

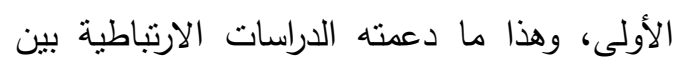

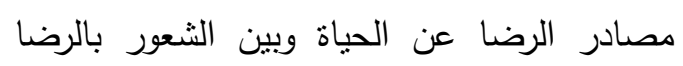
بحد ذاته، حيث أعطت هذه الدراسات نرابطات

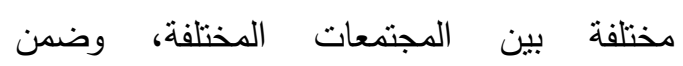
الجماعات الفرعية المختلفة مما يشير إلى إعطائها تقديرات وأوزاناً مختلفة من قبل تلك المجتمعات أو أو الجماعات ( \&eenhoven, 1991, Diener \&

.(Diener, 1995, Sam,2001

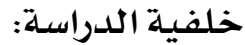
مما لا شك فيه أن شعور الفرد بالرضا (أو عدم

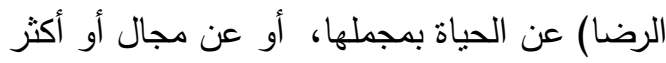

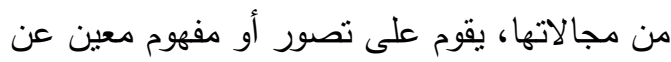
الحياة، أو جانب منها، ينتكل لادى الفرد استتاداً إلى حصيلة خبرته الثخصية وممارساته الواقعية في معترك الحياة ذاتها، ومن هذا المنظور يعكس

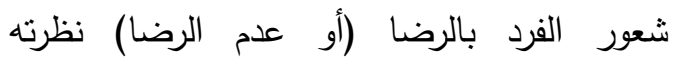
الخاصة للعالم من حوله، ونظرته لذاته على حدٍ سواء، كما يمثل حكماً أو تقويماً عقلياً معرفياً (Cognitive evaluation or judgmwnt) لنوعية الحياة التي يعيشها ككل، أو لمجالات

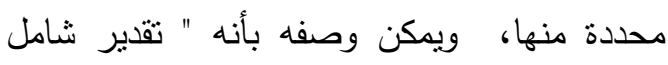

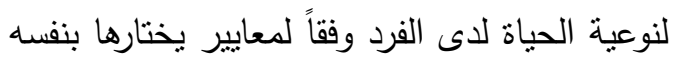
.(Shin \& Johnson, 1978: 478) ولعل مما بظهر أهمية هذا الثعور ومكانته الخاصة في حياة الفرد أنه يمثل القوة الدافعة (أونه

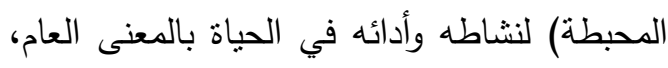
أو في هذا المجال أو ذاك من مجالاتها المختلفة، وبالتالي يسهم في عملية تكيفه الثخصي والاجتماعي، كما يطبع شخصيته بطابعها ويؤكد

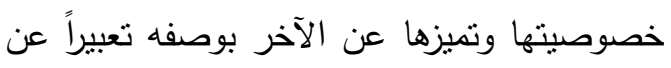
حكم عقلي معرفي يطلقه الفرد على الوجود من

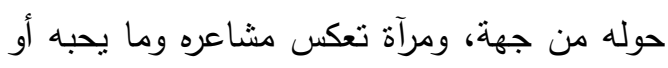

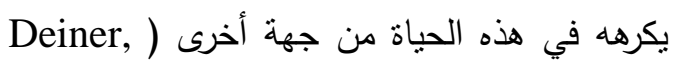
Emmons, Larsen \& Griffin, 1985, .(\&rews \& Withey, 1976

وعلى الرغم من أن اهتمامات الباحثين في مجال

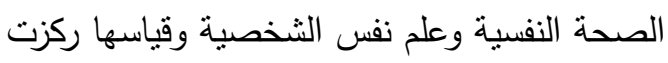
في السابق على دراسة السمات ذات الطبيعة المرضية أو السلبية للشخصية 
وتعطي درجة لكل منها على حدة؛ عمل هيوبنر على إعداد المقياس المعروف بـ " مقياس الرضا عن الحياة المتعدد ل للطلبة Multidimensional Students, Life , Satisfaction Scale كما أخضعه للعديد من الدراسات السيكومنرية التي استهدفت التحقق من ثباته وصدقه باستخدام طرائق وأساليب متعددة. وبالفعل فقد أعطى المقياس لاس موضع الاهتمام مؤشرات ثبات واتساق مرضية

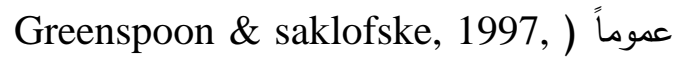
Huebner, 1994, Huebner, Laughlin, Ash \& Gilman, 1998

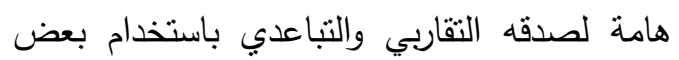

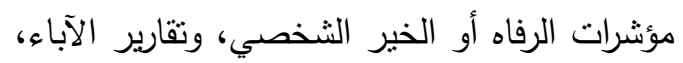

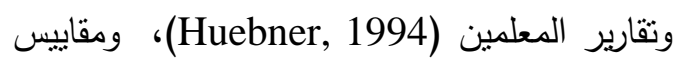

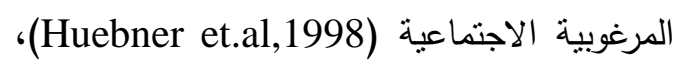
بالإضافة إلى ما سبق، دعمت التحليلات العاملية

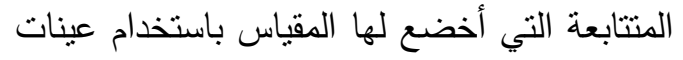
تتنمي إلى ثقافات مختلفة توزيع بنود هذا المقياس إلى خمسة مجالات فرعية، وأظهرت نوافق بنيته

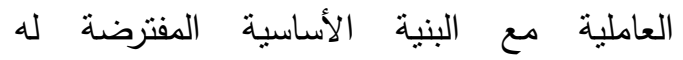
Huebner, 1994, Gileman \& Huebner, ) 2002, Huebner, et al,1998). ويشير الدليل الخاص بهذا المقياس إلى توافر كم لا بأس به من

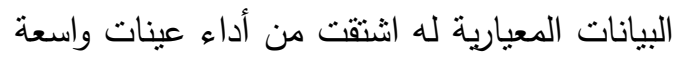
من طلبة المدارس (Huebner, 2001).

لقد أعدت الصورة المختصرة لمقياس الرضا

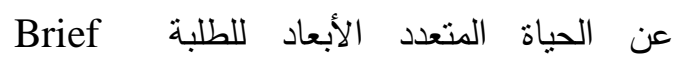
Multidimensional Students' Life Satisfaction Scale (BMSLSS) استخدامها في المسح الثشامل لسلوك المخاطرة لدى الناشئة، الذي أجراه مركز كارولينا الجنوبية لمراقبة

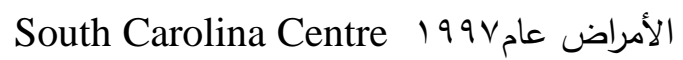
for Disease Control
لقد ظهرت أدوات عديدة لقياس الرضا عن الحياة. من أمتلة هذه الأدوات مقياس نيوجارتن Neugarten, ) وهافيغورست وطوبين Havighurst \& Tobin, 1961 المرتكز على الذات (Cantril, 1965)، ومقياس الروح المعنوية لمركز فيلادلفيا لدراسات الثنيخوخة

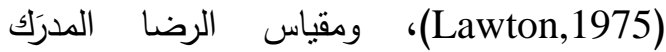
Perceived Life Satisfaction Scale هذا (Adelman,Taylor \& Nelson, 1989) بالإضافة إلى مقياس دينر ولارسن وجريفن للرضا عن الحياة ذي الثهرة الواسعة (SWLS) Satisfaction Will Life Scale يميز هذه المقاييس بمجموعها أنها اعتمدت النظرة الكلية الأحادية في تقدير الرضا عن الحياة واقتصرت على درجة كلية واحدة للتعبير عنه، وهذا لتراير ما يؤدي بنظر هيوبنر وزملائه إلى إغفال طبيعته التعددية الواسعة وطمس المجال (أو المجالات) التي قد يكون له، أو لها، الدور الحاسم في تكوين هذا الشعور وبلورته سواء بالاتجاه الإيجابي أم السلبي. ولابد أن تحل محل هذه هذه النظرة الأحادية الكلية، كما يلاحظ هيوبنر وزملاؤه، النظرة التعددية التي تتيح رصد هذا المصدر أو ذاك من مصادر

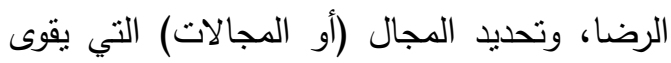
فيها هذا الثعور (سواء بالاتجاه الإيجابي أم السلبي)، وبما يتيح الكثف عن العوامل والمتغيرات Huebner,Laughlin, Ash \& ) الكامنة وراءه (Gilman, 1998 ). وتبعاً لذلك سيكون من المفيد النظر إلى مفهوم الرضا عن الحياة بوصفه مفهوماً

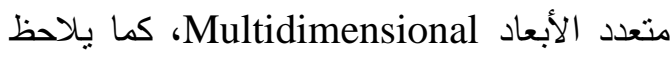

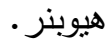

وانطلاقاً من النظرة التعددية للرضا عن الحياة وتلبية للحاجة إلى أداة قياس متعددة الأبعاد تتيح الكثف عن الأبعاد (أو المجالات) المختلفة التي يمكن أن يظهر فيها هذا الشعور بصورة منفصلة؛ 
مع نظرائها في الصورة الأم كانت أعلى بوضوح من التزابطات البينية لهذه الدقاييس سواء ضمن

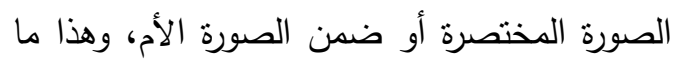
يعطي مؤشراً مهما لصدق التكوين الفرضي للصورة المختصرة (Seligson, et.al.,2003).

ومن المؤشرات المهمة الأخرى لصدق الصورة

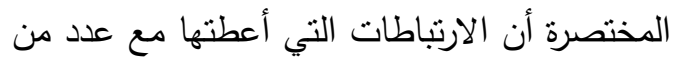
المقاييس المحكية الني تتتاول سمات مشابهة أو الون مغايرة لها جاءت بالاتجاه المتوقع لها، حيث هبطت التهات عن الحدود المتوسطة بدرجة ما، ولكن بقيت دالة. ومن أمثلة هذه الارتباطات الارتباط الذي أعطنه

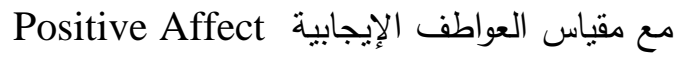
scale مقياس العواطف السلبية Negative Affect scale أعطنها مع المقاييس الفرعية الأربعة لمقياس Problem Behavior Scale السلوك المشكي بعد مرور سنتين، والتي بلغت _.r.. مع مقياس

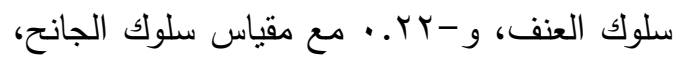

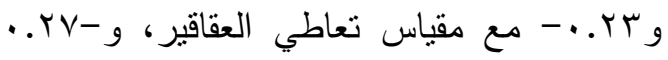
مع مقياس استفزاز الزملاء (وجميعها دالة). (Valois,Zullig,Huebner

.Drane,2001)

ومن الدراسات التي أخضعت لها الصورة الدختصرة أيضاً الدراسة الني اهتمت بالكثف عن التان

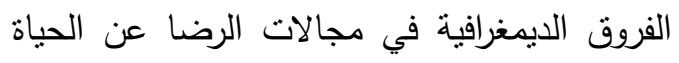
الخمسة، وفي الرضا عن الحياة بشكل عام الران

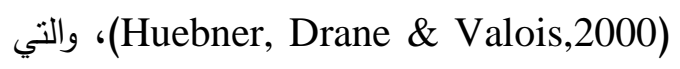
أظهرت أن الطلبة القوقازيين أظهروا درجات أعلى ألى الأل من الطلبة الأمريكان ذوي الأصول الأفريقية في الطيات الطاني

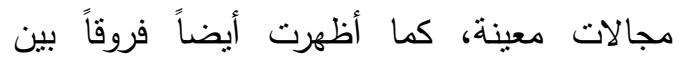

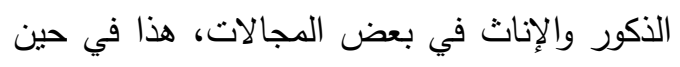
أنها لم تظهر أي فروق دالة في الرضا عن الحياة
المدارس تجاوز عددها . .00 طالباً وطالبة موزعة على با مدرسة. وتضمنت الصورة المختصرة خمسة بنود يتتاول كل منها أحد المجالات الخمسة

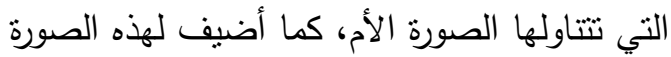
بند سادس لقياس الرضا العام عن الحياة لدى الطلبة بهدف توفير مؤشرات لصدق هذه الأداة

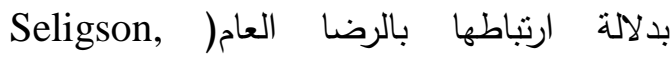
Huebner \& Valois, 2003 الصورة مستويات مقبولة للاتساق الداخلي عند استخدامها مع طلبة الصفوف الدراسية المتوسطة والعليا، حيث بلغ معامل ألفا المحسوب للارجة الكلية لهذه الصورة، والتي تمنل مجموع التقديرات

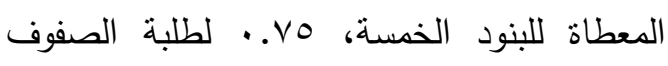

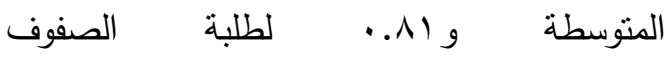
العليا(Seligson, Huebner, Valois, 2003). كما أسفر التحليل العاملي للبنود الخمسة التي

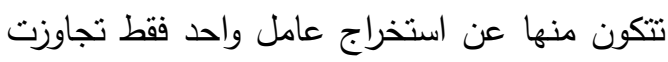

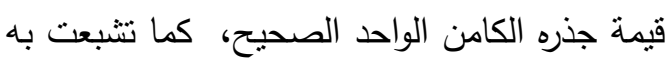

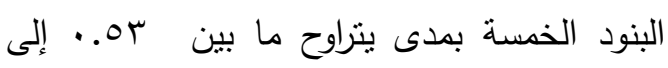

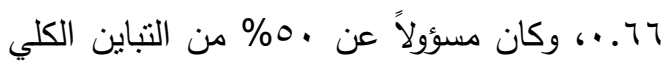

.( Huebner, Suldo \& Valois,2003 )

ومن الإجراءات التي استخدمت في دراسة صدق الصورة المختصرة مدار البحث مقارنة الترابطات التي أعطتها المقاييس الفرعية الخمسة لهذه الصورة (والتي يتكون كل منها من بند واحد التراتيط

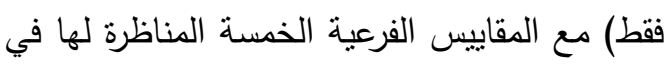
الصورة الأم (أي ارنباط كل مقياس فرعي بنظيره في الصورة الأم) بالترابطات الداخلية أو البينية

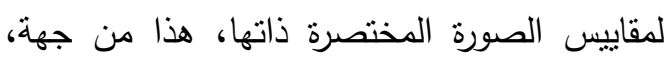
ومن جهة أخرى بالترابطات الداخلية أو البينية لمقاييس الصورة الأم ، (أي ارتباط كل مقياس فرعي بالآخر ضمن كل صورة). ولعل النتيجة الأهم التي أمكن الوصول إليها من خلا هل هذه

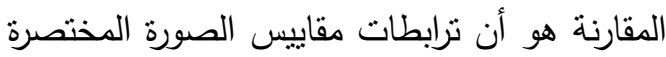


سبقت الإثارة إليه (الدسوقي،999 ()). مع ذلك فإن مسألة الرضا عن الحياة لم تحظ حتى الآن بالاهنمام الكافي في الساحة العربية، ولعل هذا

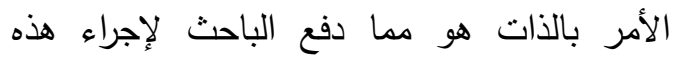
الدراسة السيكومترية للصورة المختصرة لكقياس

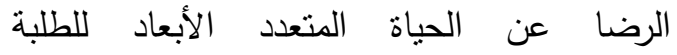
(BMSLSS) الدراسة الخاصة بالصورة الأم لهذا المقياس.

مشكلة الدراسة : - مشية يتضح من البند السابق الخاص بخلفية هذه الدراسة أن المقاييس النفسية التي تتصدى للسمات ذات الطبيعة الإيجابية للشخصية، ومنها مقاييس الرضا عن الحياة، أخذت تحظى باهتمام خاص في العيجايه

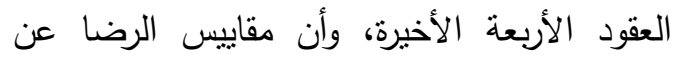

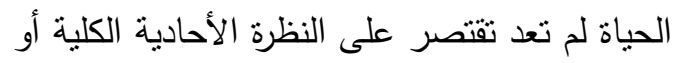

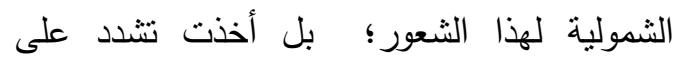

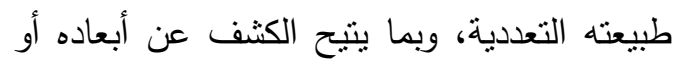

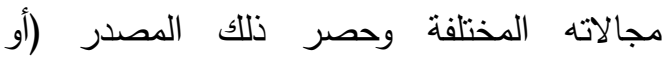
المصادر) التي قد يقوى أو يضعف فيها هذا

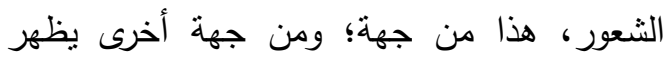
العرض السابق الافتقار إلى دراسات عربية لهذا المقياس أو لغيره من مقاييس الرضا عن الحياة، الرفان

ولعل هذا الأمر بالذات، والثعور بإمكانية الاستفادة من منل هذه الأدوات في البيئة السورية والعربية عموما هو مما دفع الباحث إلى طرح المسألة (أو الدئ

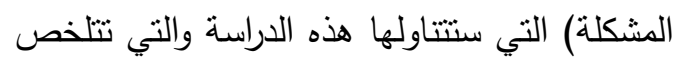
في السؤال النالي: ما الثكل الذي يمكن أن تأخذه

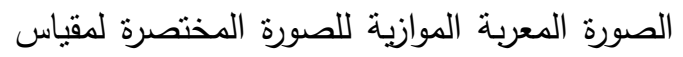
الرضا عن الحياة المتعدد الأبعاد للطلبة بعد التحقق من خصائصها القياسية (BMSLSS)

$$
\text { وصلاحها للاستخدام. بعد. }
$$

بشكل عام. وهذا ما يدعم النظرة التعددية التي ينطلق منها المقياس المتعدد الأبعاد وصورته المختصرة، ويظهر أن الاقتصار على درجة كلية

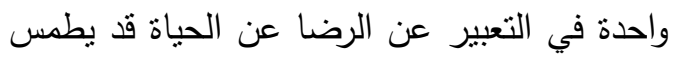
الفروق الحقيقية في مصادر (أو مجالات) الرضا بين الأفراد أو الجماعات. فقد يحصل اثثان أو أكثز ، أو جماعتان أو أكثر، على درجة " كلية" واحدة، وتظهر بينهم في الوقت نفسه اختلافات

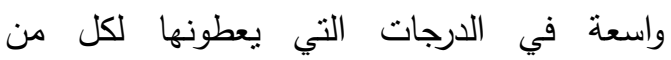
Huebner, Drane \& ) المجالات الفرعية .(Valois,2000 وبالإضافة للاراسة السابقة تجدر الإثارة إلى دراسة

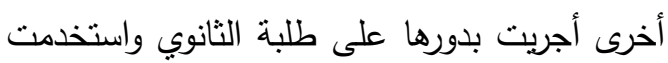

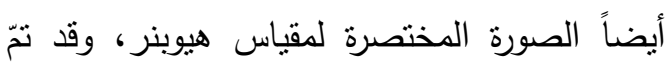
من خلال هذه الدراسة الحصول على بيانات

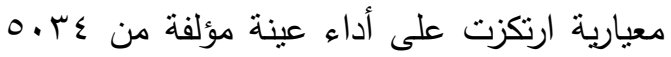

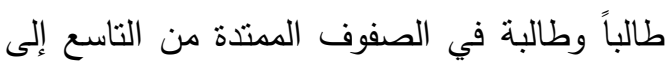
الثاني عشر من مدارس كارولينا الجنوبية، ولم تظهر هذه الدراسة فروقاً دالة يمكن أن تعزى للعرق أو الجنس أو المستوى الدراسي في الدرجة الكلية

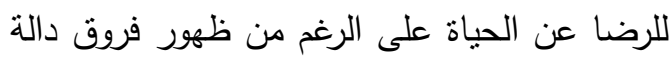
في بعض مجالات الرضا، وبذللك وفرت دعماً

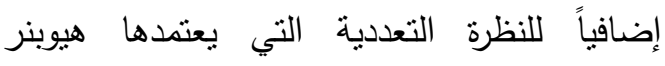
Huebner,Suldo, Valois,Drane \& ) (Zullig,2004 ومن المهر الإثارة إلى أن دراسة السمات ذات

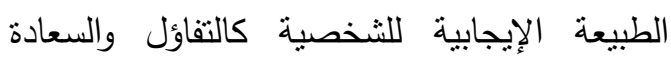
والطموح الثخصي والانبساط وغيرها أخذت تشغل مؤخراً اهتمام عدد من الباحثين العرب (مثنأ

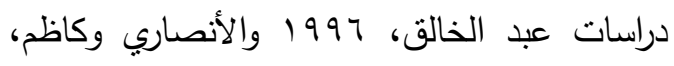
1 . . ب)، كما أجرى الدسوقي محاولة لإعداد مقياس للرضا عن الحياة استتاداً إلى بعض المض المقاييس الأجنبية ومن أبرزها مقياس دينر SWLS الذياة 
أكثر وضوحاً وتفصيلاً عن الجوانب الإيجابية في شخصية الفرد، وعن نقاط الضعف أو الصعوبات الشخصية أو الدراسية التي قد يعاني منها . r. إنها تعمل على إعداد صورة عربية موازية للصورة الأصلية المختصرة، والتحقق من إن صلاحها من خلال تطبيقها على عينات من الطلبة في المرحلة الثانوية، إضافة إلى عينات أخرى من طلبة الجامعة، وهو ما لم تخضع له الصورة الأصلية للأداة التي اقتصر تطبيقها على طلبة المرحلة الثانوية فقط، وبذلك تعمل هذه الدراسة على توفير أداة قياس جاهزة يمكن ترشيحها للاستخدام في البيئة السورية أو هاهي

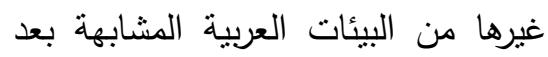
التحقق من كفاءتها السيكومترية، ومن ثم ثن الإفادة منها سواء في مجالات البحث والمقارنة بين الطلبة فرادى أوجماعات، ومن مستوى الثانوي ومستوى الجامعة على حد سواء، أو في المجالات التشخيصية والإرشادية وغيرها. r. إنها تتتاول إحدى الأدوات التي أعطت وغئه

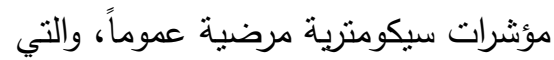
تعد أداة " اقتصادية" سهلة الاستعمال للغاية، حيث بستغرق تطبيقها حوالي

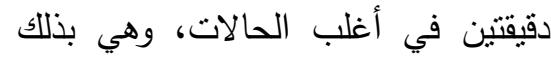
تماثي الاتجاه الجديد في تنوير المقاييس واستخدامها والذي ينطلق من ضرورة " مواءمتها" لعصر السرعة واختزال الوقت اللازم لإجرائها إلى الدرجة
هدف الدراسة وأسئلتها:

يتلخص الهذف الرئيس لهذه الدراسة في التحقق من الكفاءة السيكومترية لصورة العربية الموازية المختصرة لمقياس الرضا عن الحياة المتعدد الأبعاد للطلبة BMSLSS ويمكن التعبير عن هذا الهذف الهف

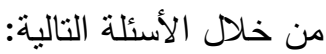
ا. ما معاملات ثبات الاستقرار والاتساق للصورة المختصرة المعربة لمقياس الرضا لإنال عن الحياة المتعدد الأبعاد للطلبة. r. ما مؤشرات الصدق التقاربي والتباعدي التي تعطيها الصورة المختصرة المعربة للمقياس المذكور بدلالة عدد من المقاييس

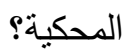
r. ما مؤشرات الصدق البنيوي (أو صدق التكوين الفرضي) التي توفرها الترابطات البينية أو الداخلية Intercorrelations للمقاييس الفرعية الخمسة للصورة المختصرة المعربة للقياس المذكور، لمعله والترابطات المحسوبة بين كل مقياس فرعي لهذه الصورة ونظيره في الصورة الأم، إضافة إلى ارتباط هذا المقاييس

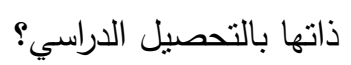

$$
\text { أهمية الدراسـة : }
$$

تظهر أهمية هذه الدراسة فيما يلي :

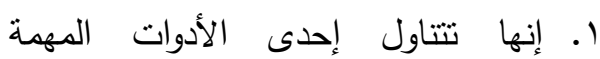

الخاصة بقلياس السمات الإيجابية

للشخصية وهي : مقياس الرضا عن الحياة المتعدد الأبعاد للطالب بصورته المختصرة BMSLSS، هذا المقياس الذي ينطلق من نظرة تعددية للرضا عن الحياة تتيح حصر المجال (أو المجالات) لهن لرصنات التي قد يقوى أو يضعف فيها هذاء لتهال الشعور، وبالتالي تسهم في إعطاء صورة 


$$
\text { منهجج الدراسـة : }
$$

لا يخرج المنهج المستخدم في هذه الدراسة عن

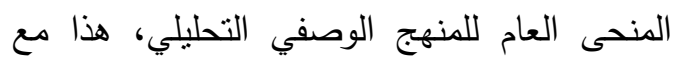
الإشارة إلى أنه تلبية لأهداف هذه الدراسة ومراعاة

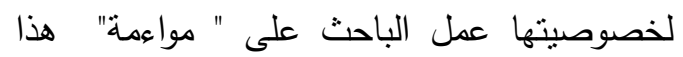
المنهج لطبيعة المشكلة موضع البحث، وبما يلبي الشروط التي تتطلبها الدراسة السيكومترية لأداة البحث، وعملية التحقق من كفاءتها القياسية.

\section{مجموعات الدراسـة :}

أُجريت هذه الدراسة على مجموعات عدة من من

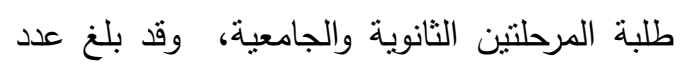

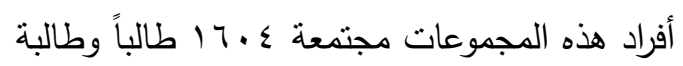
( ) (

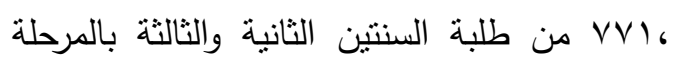
الجامعية )، ويظهر الجدول (1) توزية الجنع أفراد المجموعات المدروسة وفق الغرض الخاص بكل بكل دراسة من الدراسات التي استهدفت بمجموعها التحقق من الكفاءة القياسية للصورة المختصرة المعربة .

حدود الدراسة :

ما من شك في أن الأداة المستخدمة في هذه

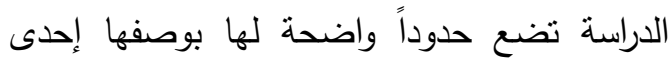
الأدوات التي تستخدم نهج التقرير الذاتي، وتعتمد كلياً على ما يقوله المبحوث عن نفسه بغض النظر عن صحته ومطابقته (أو عدم مطابقته) للواقع، هذا

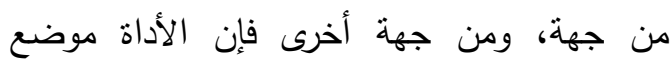
الاهتمام يفترض أن تغطي الأبعاد (أو المجالات)

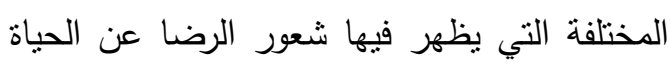

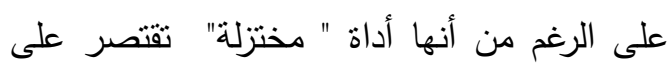
خمسة بنود فقط، وهذا ما يضع بدوره حدوداً لهذه الها

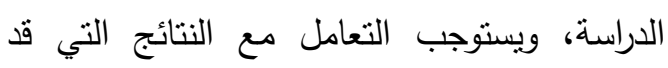
تسفر عنها بمنتهى النأني والحذر . ومن الواضح أن مما يضع حدوداً لهذه الدراسة

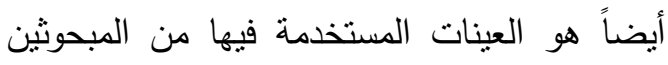

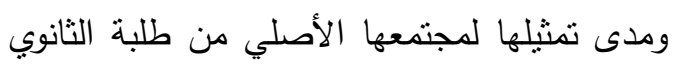

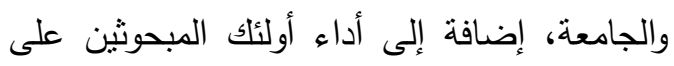

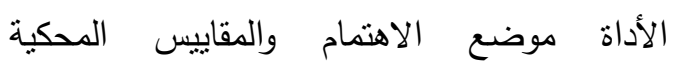
المستخدمة في دراستها في الفترة التي طبقت فيها والتي امتدت من بداية الفصل الأول من العام

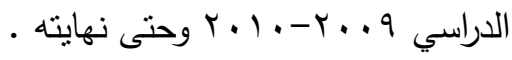

الجدول (1):

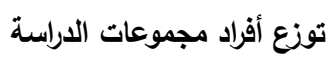

\begin{tabular}{|c|c|c|c|c|c|c|}
\hline مجموع ن & مجموع & (مجموعات مختلطة) & الإناث & الذكور & المستوى الدراسي & (الإحصائي المحسوب) البيانات) \\
\hline \multirow{4}{*}{ 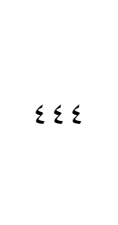 } & זr & - & $\pi$ & or & الأول الثانوي & \multirow{4}{*}{ حساب معاملات ثبات الاستقرار والاتساق } \\
\hline & & & Tr & or & الثاني الثانوي & \\
\hline & $r$ & - & 71 & $\varepsilon \varepsilon$ & الثانية جامعة & \\
\hline & & & r & $r v$ & الثالثة جامعة & \\
\hline \multirow{7}{*}{797} & & Tr & & & & \multirow{7}{*}{ والتباعدي والتزابطات البينية معاملات الصدق التقاربي } \\
\hline & & V4 & & & & \\
\hline & r4. & $\wedge \mathrm{T}$ & - & - & الثاني الثانوي & \\
\hline & & 71 & & & & \\
\hline & & TV & & & & \\
\hline & \multirow[t]{2}{*}{ צחיז } & vr & - & - & \multirow[t]{2}{*}{ الثانية جامعة } & \\
\hline & & 70 & & & & \\
\hline
\end{tabular}




\begin{tabular}{|c|c|c|c|c|c|c|}
\hline مجموع ن & مجموع & (مجموعات مختلطة) & الإناث & الأكور & المستوى الدراسي & $\begin{array}{c}\text { (الغرض من البيانات } \\
\text { (الإحصائي المحسوب) }\end{array}$ \\
\hline & & $\begin{array}{l}4 t \\
\text { Ri } \\
4\end{array}$ & & & & \\
\hline TYE & TYE & - & 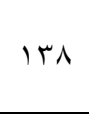 & 14 & الثانية جامعة & والتباعدي (بدلابلة معاملات آيزنك) \\
\hline$r \leqslant$. & $r \varepsilon$. & - & $11 \pi$ & IrV & الثاني الثانوي & 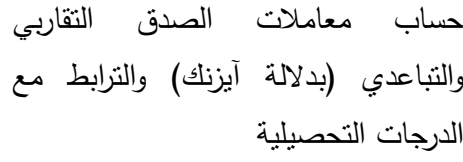 \\
\hline $17 . \varepsilon$ & 17.8 & 797 & 0.0 & $\varepsilon \cdot r$ & & الدجموع \\
\hline
\end{tabular}

r. مقياس الرضا عن الحياة للطلبة

Student Life

(SLSS)

Satisfaction Scale مؤلف من r Y بنداً أعده هيوبنر عام

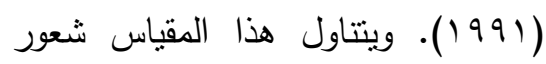
الرضا العام عن الحياة لدى الطلبة تحديداً، كما يعبر عن هذا الثعور من خلال درجة كلية واحدة فقط بخلاف لفيل المقياس المتعدد الأبعاد للطالب وصورته

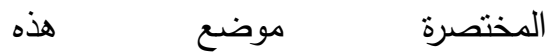
Huebner ,1991, Huebner, الدراسة هوضعة 1995). وقد قام الباحث بتعريب هذا

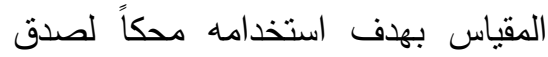

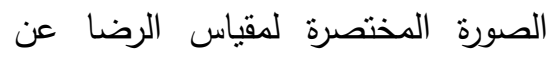
الحياة المتعدد الأبعاد للطلبة موضع لرئس لرضية الدراسة .

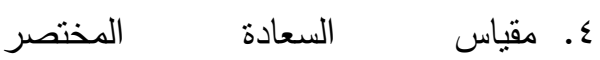
Affectometer 2 للفرح أو السعادة أعدّه كامان وفليت (Kamman\&Flett,1988). الباحث بتعرييه بهدف استخدامه محكاً لمقياس الرضا عن الحياة المتعدد الأبعاد للطلبة موضع الدراسة. ه. مقياس الدافع للإنجاز للأطفال والراشدين : وهو من إعداد هيرمانز Hermanes،

$$
\text { أدوات القياس المستخدمة يِّ الدراسـة : }
$$

من أجل التحقق من الصدق التقاربي والتباعدي للصورة المختصرة المعربة مجال البحث الحالي؛ عمد الباحث إلى استخدام خمسة مقاييس محكية معربة، كما أنه استخدمها هي ذاتها في دراسته

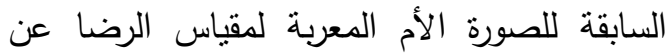

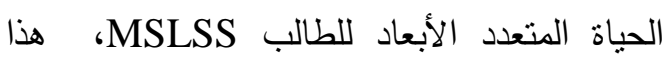
بالإضافة إلى محك سادس اعتمد في هذه الدراسة وهو مقياس الانبساط والعصابية لآيزنك (الصورة

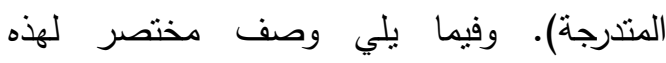
المقاييس :

ا. مقياس الرضا عن الحياة (SWLS)

:Satisfaction With Life Scale أعدّ هذا المقياس من قبل دينر وزملائه (Diener et.al.,1985) تقدير عام شامل لنعور الرضا عن الحياة بغض النظر عن المجالات المختلفة التي

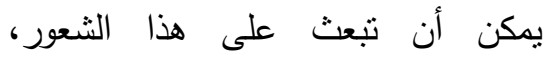
وبالتالي فهو يقتصر على بلى r. درجة كلية واحدة للتعبير عن هذا لتهئ الثعور، وقد قام الباحث بتعريب هذا المقياس، كما أخضعه للإراسة السيكومترية . 
ا. قام الباحث في المرحلة الأولى بإعداد ترجمة أولية لبنود هذه الأداة وتعليماتها، كما أوكل مهمة القيام بإعداد ترجمة ثانية لهذه الأداة لزميل له مختص باللغة الإنكليزية دون إطلاعه على الترجمة التي لهاني أعدها، وبالانتهاء من ذلك قام الباحث

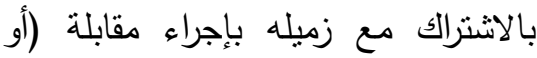
مقارنة) بين الترجمة التي أعدها كل لإنل منهما والأصل، وهذا ما أناح اختيار

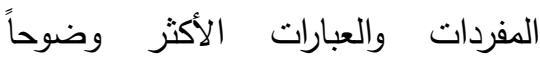
وانطباقاً مع الأصل، سواء من البنود أم التعليمات، و ومن ثم الوصول إلى صورة أولية معربة واحدة للأداة. r. عرضت الصورة الأولية المعربة للأداة ومعها النص الأصلي على اثثين من أساتذة اللغة الإنكليزية في جامعة دمشق الصفي من أجل إخضاعها للتحكيم، ومع أن هذا التحكيم لم يؤد إلى أي تغيير في الترجمة إعها المقترحة فقد عزز الثقة بالصورة المعربة لئد المقترحة للأداة.

r. تركز العمل في المرحلة الثالثة في إجراء ما يعرف بـ " الترجمة العكسية للأداة") Back Translation المهمة مشكوراً أحد أساتذة اللغة الإنكليزية بجامعة دمثق، وهو الذي قام بترجمة

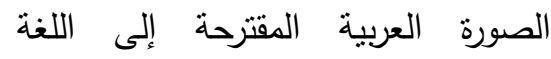

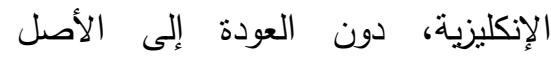

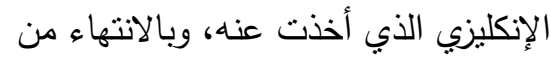
هذا العمل قام الباحث بالاشتراك مع زميله

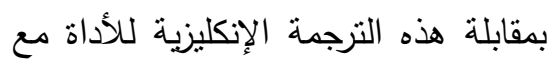
الأصل الإنكليزي، وقد وفرت هذه المقابلة المزيد من الثقة بالترجمة العربية المقترحة للأداة، حيث ظهر نوافق يكاد يصل إلى لى لئل حد النطابق بين الترجمة الإنكليزية
ويقيس الدافع للإنجاز للى الأطفال والراشدين على حد سواء، وقد قام موسى بتعريبه، ونشر كراسة التعليمات الخاصة به (موسى، (9191) ). 7. Beck ) :Depression Inventory(BDI وضع الصيغة المعدلة لهذا المقياس بيك R.A.Steer وستير A.T.Beck ب991 ـ وقام بإعداد الصورة العربية لهذا المقياس عبد الخالق من جمهورية مصر

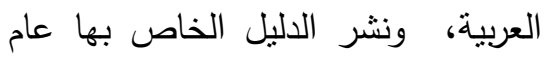

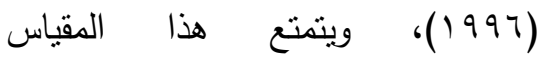
بخصائص قياسية جيدة سواء في صورته

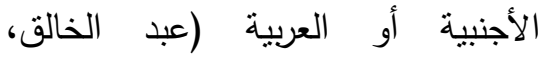
.$(1997$ V. مقياس الانبساط والعصابية (الصورة المتدرجة): وهو يتتاول بعدي الانبساط والعصابية فقط من الصورة القصيرة لبنائ لمقياس آيزنك للشخصية EPQR-S، ويقتصر بذلك على \& ب بنداً من أصل ^) بنداً تتضمنها تلك الصورة، وقد قام الباحث بإعداد بتعريبه، كما أخضعه لبها

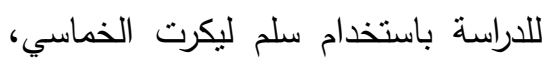
وذلك في إطار المشروع البحثي الثامل الذي نفذه لصالح جامعة دمشق عام إملام

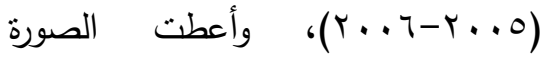
المعربة لهذا المقياس مؤشرات سيكومترية مرضية.

إعداد الصورة المعربة الموازية لصورة المختصرة وإجراءات التطبيق:

تطلبت عملية إعداد الصورة المعربة موضع البحث المرور بعدة مراحل وخطوات،هي: 
بصلاحيتها وإمكانية استخدامها مع طلبة الثانوي والجامعة على حد سواء.

7. وبالاتتهاء من إعداد الصورة المعربة

للأداة بشكلها النهائي ثم الانتقال إلى إنى مرحلة الدراسة السيكومترية لهذه الصورة، وقد تطلبت هذه الدراسة تطبيق الصورة المعربة المقترحة على مجموعات عديدة من طلبة الثانوي والجامعة، كما تطلبت تطبيق الصورة المعربة الأم لهذه الأداة

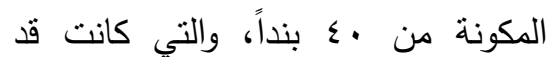
أعدت في دراسة سابقة للباحث نفسه، إضافة إلى ستة مقاييس محكية اعتمدها

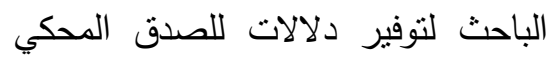

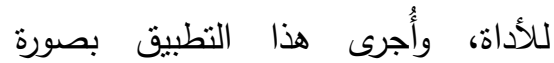
جماعية داخل كل قاعة دراسية على حدة لهداه ودون فرض حدود زمنية على المبحوثين،

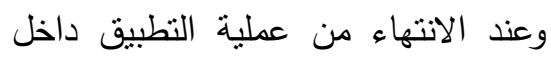
كل قاعة كانت أوراق الإجابة (بما فيها أوراق الإجابة عن المقاييس المحكية

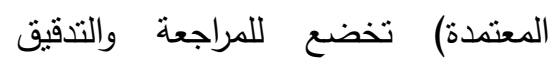

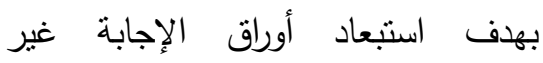
المكتملة أو الفارغة أو التي اعتمداد الإجابه الإجابة العشوائية ( وقد بلغ عددها بارها ورقة )، وقد تم في خطوة لاحقة إدخال البيانات إلى الحاسوب لتخضع للمعالجة الإحصائية وفق الأهداف الخاصة بهذه الدراسة، وتظهر في الملحق (1) الصورة المعربة للأداة بشكلها النهائي.

دلالات الثبات والصدق للصورة المختصرة المعربة (النتائج ومناقشتها): ا. النتائج الخاصدة بالسؤال الأول : للإجابة عن السؤال الأول من أسئلة هذه الدراسة

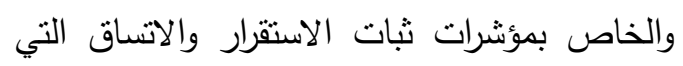

والأصل فيما يخص التعليمات(أو الإرشادات) وثلاثة بنود، إلا أنه ظهرت فروق طفيفة بينهما في الصباغة التعبيرية للبندين الثالث والخامس، وهي لا تؤثر في المعنى الدقيق لكل منهما . المان ع. قام الباحث في المرحلة الرابعة بتطبيق الأداة المختصرة بصورتيها الإنكليزية والمعربة على مجموعة من الأفراد يمكن الادداة وصفهم بأنهم مزدوجو اللغة Bilingual، وبحيث يأخذ كل منهم إحدى الصورتين تليها الأخرى، وقد تكونت هذه المجموعة من 91 طالباً وطالبة من طلبة السنة الرابعة من قسم اللغة الإنكليزية بجامعة من طنة دمشق، وبلغت معاملات الارتباط المحسوبة بين كل من المقاييس الفرعية الخمسة للصورة العربية للأداة ونظيرتها في 97 . •لمقياسي الأصدقاء و والمدرسة، و90. . لقياس الذات، ع 9. . ل لمقياس ولاس

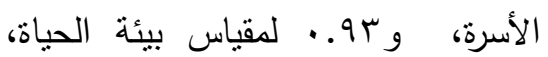
ومع مراعاة حقيقة أن ضآلة عدد البنود التي تتكون منها الأداة يمكن أن تكون قد اند

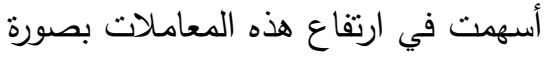

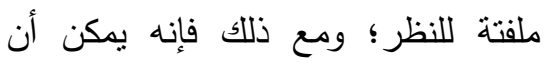
تؤخذ دليلاً آخر حول تعادل الصورتين العربية والإنكليزية. ه. طبقت الصورة المعربة على مجموعات استطلاعية من طلبة الثانوي والجامعة

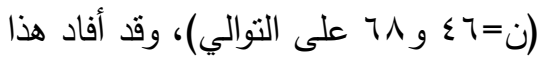
التطبيق في التأكد من وضوح بنود هذه

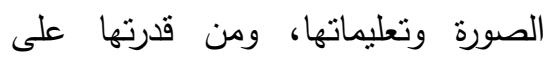
استثارة دافعية المبحوثين وتجاوبهر معها، وحسن إخراجها، وذلك وفّر مؤشراً للصدق الظاهري لهذه الصورة، كما وفر النقة 
وإناث من طلبة السنتين الثانية والثالثة )، كما أعيد تطبيقها ثانية على المجموعات ذاتها بعد مرور فواصل زمنية معينة، وهذا ما يظهر في الجدول
يمكن أن تعطيها الصورة المختصرة المعربة مجال البحث الحالي طبقت هذه الصورة على مجموعات

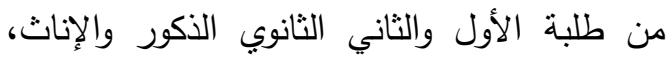
ومجموعات أخرى من طلبة كلية التربية (ذكور

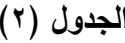

معاملات ثبات الإعادة للصورة المختصرة لمجموعات من طلبة الثانوية والجامعة

\begin{tabular}{|c|c|c|c|c|c|c|c|c|}
\hline \multicolumn{4}{|c|}{ طلبة الجامعة } & \multicolumn{4}{|c|}{ طلبة الثانوي } & \multirow{3}{*}{ الفرعية } \\
\hline \multicolumn{2}{|c|}{ الفاصل : • ד يوم } & \multicolumn{2}{|c|}{ الفاصل : ؛ ايوم } & \multicolumn{2}{|c|}{ الفاصل : • ד يوم } & \multicolumn{2}{|c|}{ الفاصل : ؛ ايوم } & \\
\hline $\begin{array}{c}r \\
(\dot{l})\end{array}$ & 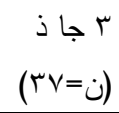 & \begin{tabular}{c}
$r$ \\
\multicolumn{1}{l}{$(r \Lambda=(ن)$}
\end{tabular} & 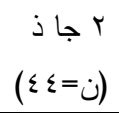 & 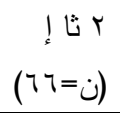 & 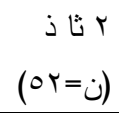 & $\begin{array}{c}\text { أثا } \\
(\pi r=0\end{array}$ & 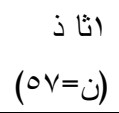 & \\
\hline. $.7 V$ & $.7 \varepsilon$ & $\cdot . V r$ &.$v 1$ & - . &. .70 & אז.. &. .79 & الأسرة \\
\hline .71 &. .79 &.$V \varepsilon$ &. .79 & $.0 r$ & .77 & $\cdot V \varepsilon$ &.$v 1$ & الأصدقاء \\
\hline$\because v$. & .71 & $\cdot \vee \wedge$ &.$V r$ & .71 & $.7 \mathrm{~V}$ & $\cdot v 1$ &.$V Y$ & المدرسة \\
\hline. .70 &. .79 &. .79 & $\because V \varepsilon$ & $\because v$. & .71 &.$V Y$ &. .77 & بيئة الحياة \\
\hline. $.0 \mathrm{~V}$ &.$V r$ & $\cdot v \cdot$ & $\cdot v \varepsilon$ & $\cdot . \leqslant \Lambda$ & $.0 r$ &. .79 &. .71 & الذات \\
\hline
\end{tabular}

أسبوعين بلغ اV. . في حين أن وسيط المعاملات

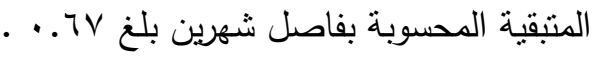
أما المعاملات المحسوبة لطلبة الجامعة فقد

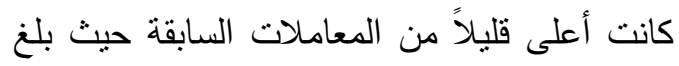
وسيط المعاملات المحسوبة بفاصل أسبوعين

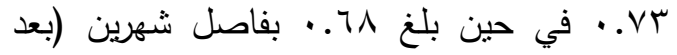
استبعاد معامل واحد فقط أعطاه مقياس الذات وبلغ

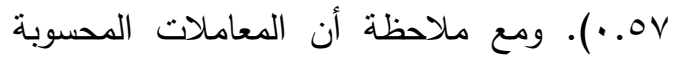
بفاصل شهرين أظهرت هبوطاً عن ثلاك المحسوبة بفاصل أسبوعين، وهو أمر منوقع ومنتظر، فإن النتائج السابقة بمجموعها يمكن أن تعطي مؤشراً لثبات الصورة المختصرة المعربة التي ذُرست بطريقة الإعادة (أو ثبات الاستقرار) لدى طلبة الثانوي والجامعة على حد سواء، هذا مع الإثارة إلى أن ظهور شيء من الهبوط الطفيف في مؤشرات ثبات الاستقرار لدى طلبة الثانوي بالمقارنة

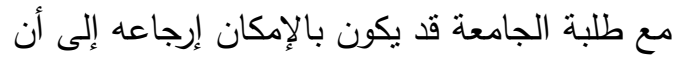

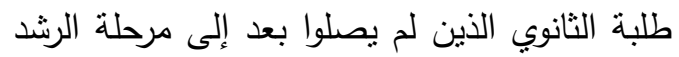

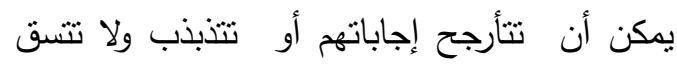

ويظهر الجدول (Y) أن معاملات الثبات المحسوبة بطريقة الإعادة اقتربت من الحدود

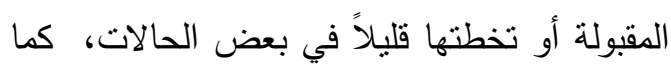

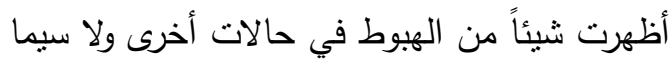
لدى طلبة الثانوي، كما يظهر هذا الجدول فروقاً

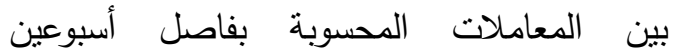
والمعاملات المحسوبة بفاصل شهرين (لصالح الأولى)، وهو ما ينسجم مع التوقعات، وفروقاً أخرى بين المعاملات المحسوبة لطلبة الثانوي والمعاملات المحسوبة لطلبة الجامعة لصالح الثانية)، ويتبين من قراءة المعاملات المحسوبة

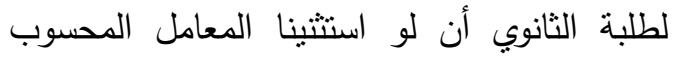

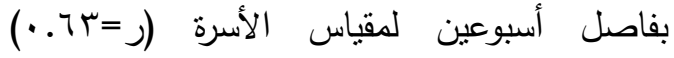
والمعاملات الثلاثة المحسوبة بفاصل شهرين

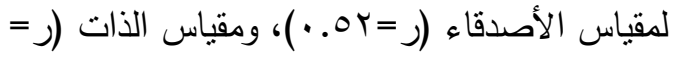

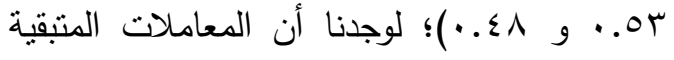

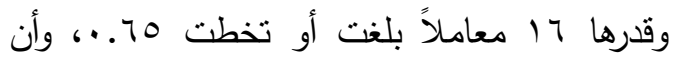
وسبط المعاملات المتقية المحسوبة بفاصل 
موضع الدراسة أن تحقق درجة عالية من التجانس

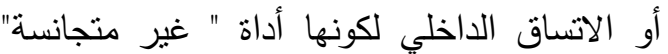
أساساً تشدد على النظرة التعددية بدلاً من النظرة الأحادية الكلية للرضا عن الحياة، هذا بالإضافة إلى أنها أداة مختصرة تقتصر على خمسة بنود، ومع ذللك فإن النتائج السابقة والخاصة بثنات الاستقرار والاتساق للصورة المعربة يمكن وصفها

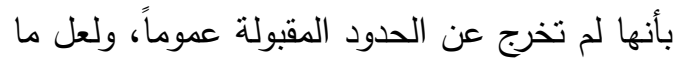
يضفي على هذه النتائج شيئًاً من الأهمية هو أنها

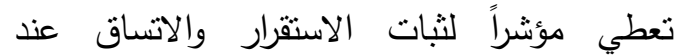

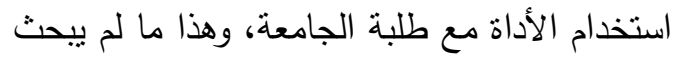
من فبل في الدراسات الأجنبية للصورة الأصلية للأداة والتي اقتصرت على طلبة المرحلة قبل

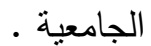

\section{r- النتائج الخاصة بالسؤال الثاني:} للإجابة عن السؤال الثاني من أسئلة هذه الدراسة

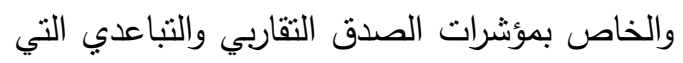
بمكن أن تعطيها الأداة مجال البحث طبقت الأداة كما طبقت معها خمسة مقاييس محكية معربة كان الباحث قد استخدمها في دراسة سابقة في استخراج بعض دلالات الصدق التقاربي والتباعدي للصورة

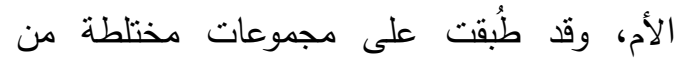

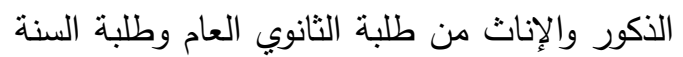
الثانية في كلية الآداب في جامعة دمشق (قسم علم اجتماع). وتظهر في الجدول (r) النتائج الخاصة

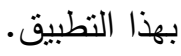

مع نفسها بدرجة أكبر من الراثدين، وذلك بفعل المرحلة العمرية التي يمرون بها، وهي مرحلة المراهقة المتأخرة التي تتسم بالكثير من الاضطراب وعدم الاستقرار النفسي والعاطفي.

وبالإضافة إلى طريقة الإعادة، حسبت معاملات

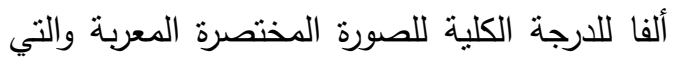
تمنل مجموع التقديرات المعطاة للبنود الخمسة التي لني

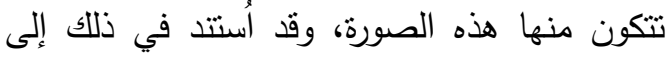
أداء أفراد المجموعات السابقة نفسها من طلبة لاهية الثانوي والجامعة، وبلغت قيم هذه المعاملات ب V . .

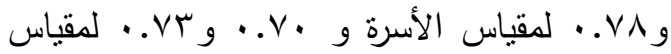
الأصدقاء و 71.. و وVV.. لمقياس المدرسة، و 79.. و وVY.. لمقياس بيئة الحياة، و وكV. و^ף.. لمقياس الذات لطلبة الثانوي وطلبة الجامعة على التوالي، وبذلك جاءت هذه المعاملات في مدى يتراوح ما بين \^.

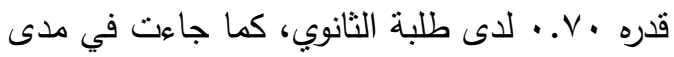

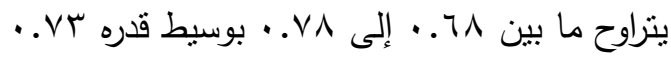

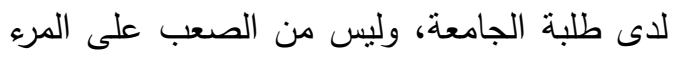
أن يلحظ أن معاملات ألفا السابقة يمكن أن تشير لتهري إلى درجة مقبولة من الاتساق الداخلي للصورة المعربة مجال البحث الحالي، على الرغم من أنها

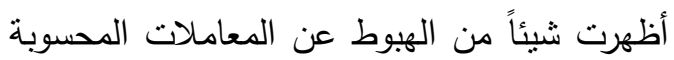
والمناظرة لها للصورة الإنكليزية المختصرة(والتي بلغ الغن وسيطها ه v.. لطلبة الصفوف المتوسطة و اN. .

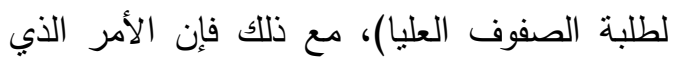
يجدر أخذه في الحسبان هو أنه لا يتوقع للأداة 


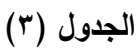

معاملات الارتباط للصورة المختصرة المعرية مع عدد من المقاييس المحكية

لعينات من طلبة الثانوية والجامعة

\begin{tabular}{|c|c|c|c|c|c|c|c|c|c|c|}
\hline \multicolumn{2}{|c|}{ بيك للاكتئاب } & \multicolumn{2}{|c|}{ الدافع للإنجاز } & \multicolumn{2}{|c|}{ السعادة المختصر } & \multicolumn{2}{|c|}{ الرضا عن الحياة } & \multicolumn{2}{|c|}{ الرضا عن الحياة } & \multirow{2}{*}{ المقاييس } \\
\hline $\begin{array}{c}\text { Y } \\
\left(\begin{array}{l}V= \\
(Y)\end{array}\right.\end{array}$ & $\begin{array}{c}\dot{H} r \\
(T V=\dot{Y})\end{array}$ & 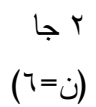 & 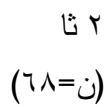 & $\begin{array}{r}r \\
\text { r }\end{array}$ & 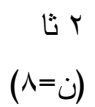 & 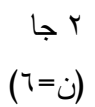 & 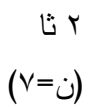 & 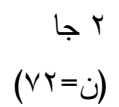 & 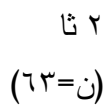 & \\
\hline..$\leqslant \Gamma_{-}$ & $\ldots v-$ & אז.. & ו &.$\mu V$ & ו &. .01 & $\cdot . \leqslant 9$ &..$\leqslant 0$ & .01 & الأسرة \\
\hline$. r V-$ & $. .1 \leq-$ & $\cdot . \leqslant \wedge$ &.$r V$ &.$r \varepsilon$ &..$r T$ &. .09 &. .01 & $\cdot . \leqslant 9$ & $\therefore \leqslant V$ & الأصدقاء \\
\hline . TO- & - & . & 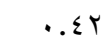 & .. &. $.4 q$ &. .00 & אד. &..$\leqslant \varepsilon$ & $\cdots \leqslant 9$ & المدرسة \\
\hline$. r V-$ & $. r \wedge-$ & ( &.$r V$ & .ro &..$r \wedge$ & $\cdot . \leqslant V$ & . & (. & וM & بيئة الحياة \\
\hline - דז". & .rq- &..$\leqslant \varepsilon$ & .. & דצr. & דצr. & .00 & $.0 r$ & .. &.$r q$ & الذات \\
\hline - & $. r \wedge-$ &..$\leqslant \varepsilon$ & ו ו . & דr &. rA &. .00 & .04 &..$\leqslant \varepsilon$ &..$\leqslant V$ & الوسيط \\
\hline
\end{tabular}

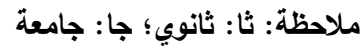

الننائج الني أسفرت عنها دراسة الصورة النركية المختصرة للأداة مجال البحث بدلالة محك بيك النكان للاكتئاب والني أعطت بدورها ارتباطات سالبة لإنة أكثرها دالة (Digden,2008)، كما يذكرّ بالنتيجة النيان التي أعطاها مقياس دينر للرضا العام عن الحياة

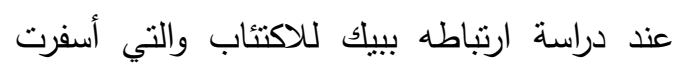

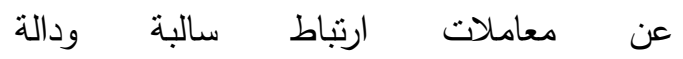

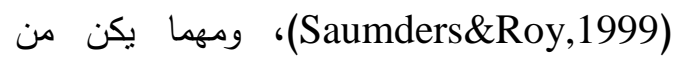

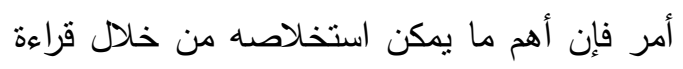

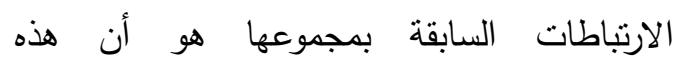

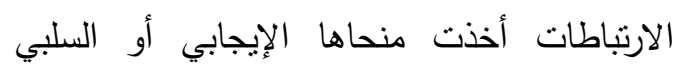

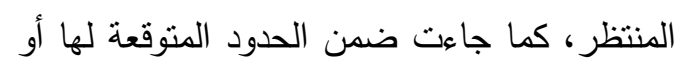
أنها، في أسوأ الأحوال لم تخالف الثوقعات المرتقبة الماتية

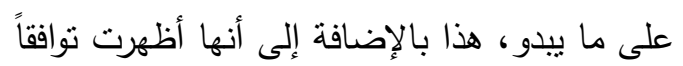
ملحوظاً لاى طلبة الثانوي والجامعة (باستثاء الارتباطات المنحصلة مع مقياس الداقع للإنجاز

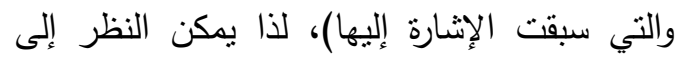

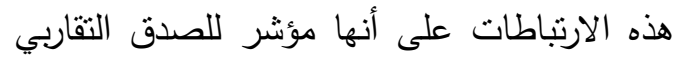

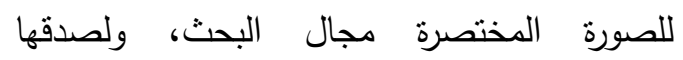
التباعدي على حذ سواء، ذلك لأنها أظهرت درجات

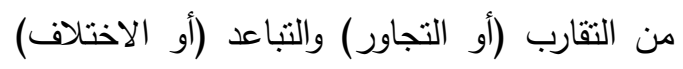

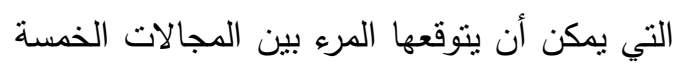

يتبين من قراءة الجدول (ץ) أن الارتباطات التي

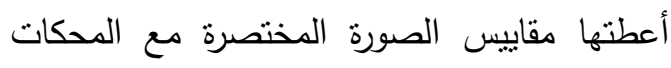
المعتدة اقتربت من الحدود المتوسطة، كما ابتعدت العتان عنها أحياناً بدرجة ما، ولكنها كانت دالة (باستثناء

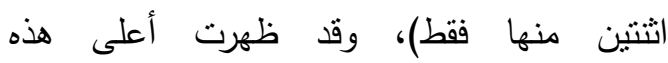
الارتباطات مع مقياس الرضا عن الحياة للطلبة الطان

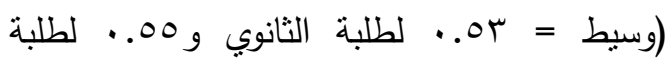
الجامعة )، يليه مقياس الرضا العام عن الحياة

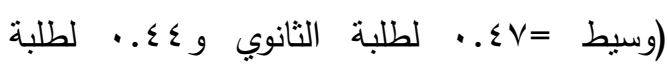

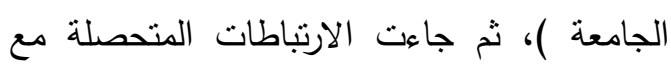

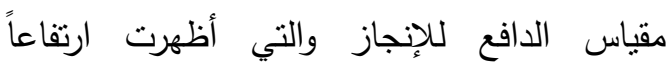

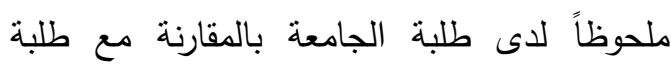

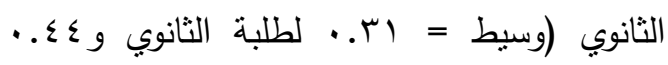

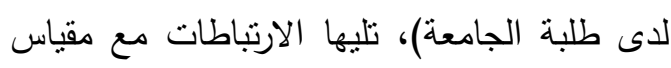

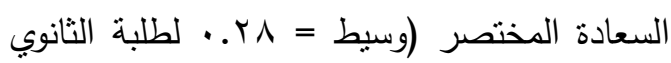
و وr.r. لاى طلبة الجامعة ). هذا في مقابل

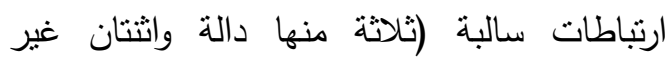

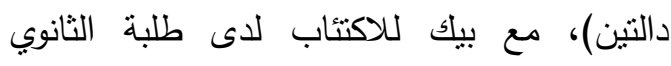

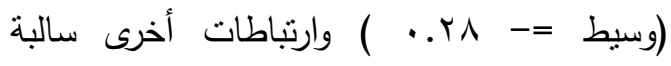

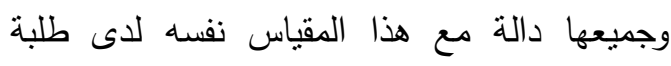

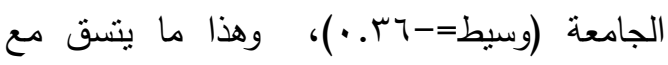



والعصابية. وارتكزت هذه الدراسة على أداء مجموعة من طلبة الصف الثاني الثانوي العام الذكور والإناث وطلبة السنة الجامعية الثانية الذكور والإناث (تخصص طب الأسنان).وتظهر النتائج

الخاصة بها في الجدول (ع).
وهو الصورة المتدرجة لمقياس آيزنك للانبساط

للرضا عن الحياة لدى الطالب من جهة؛ وبين شعور الرضا العام عن الحياة أو متغيرات ومظاهر

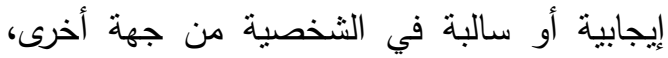
كالدافع للإنجاز ، والسعادة، والاكتئاب.

ودراسة الصدق النقاربي والتباعدي للصورة المختصرة موضع البحث لم تتوقف عند الحدود

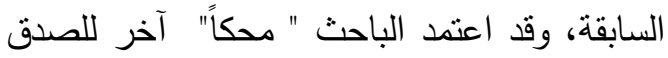

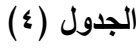

ترابطات الصورة المختصرة المعرية مع الانبساط وإلعصابية

\begin{tabular}{|c|c|c|c|c|c|c|c|c|}
\hline \multicolumn{4}{|r|}{ العصابية } & \multicolumn{4}{|r|}{ الانبساط } & \multirow[b]{2}{*}{ المقاييس الفرعية } \\
\hline 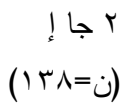 & 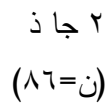 & 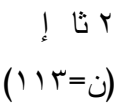 & 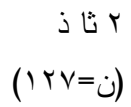 & 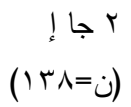 & 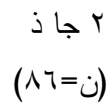 & 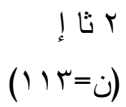 & 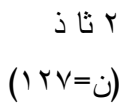 & \\
\hline$\cdot .9-$ & I. & $\begin{array}{r}- \\
* * . r q\end{array}$ & $* . . r \leqslant-$ & *..r & **. r & $* . r T$ & $* * . r q$ & الأسرة \\
\hline $\begin{array}{r}- \\
* * . r \leq\end{array}$ & $\begin{array}{r}- \\
* \% \text {. TV }\end{array}$ & $\ldots \cdot 1-$ & 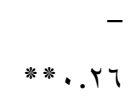 & **.r. & $* *$. ro & $\cdots v$ & $* . . Y Y$ & الأصدقاء \\
\hline 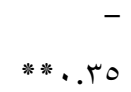 & $. .1 \%-$ & $\begin{array}{r}- \\
* * . \Gamma \leq\end{array}$ & $\begin{array}{r}- \\
* * \text { * }\end{array}$ & $* * . . \leq r$ & ***. & **.. Y Y & **. & المدرسة \\
\hline $.11-$ & **.. & 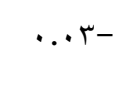 & $* . r M-$ &...$r$ & $* * . . Y q$ & $* * . \mu 1$ & שT. • & بيئة الحياة \\
\hline *..rT- & $\cdots \varepsilon-$ & **. & $. . r-$ &. .11 & $* * . \mathrm{rV}$ & $* . . Y T$ & $* * . . Y q$ & الذات \\
\hline
\end{tabular}

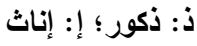

ملاحظة: ثا: ثانوي؛ جا: جامعة

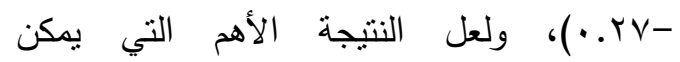
استخلاصها هنا أيضاً هو أن هذه الارتباطات الاته

أخذت منحاها الإيجابي المنتظر فيما يتصل بمقياس الانبساط، كما أخذت منحاها السلبي المنتظر فيما يخص مقياس العصابية، ولم تخرج على ما يبدو عن الحدود المتوقعة لها في أغلب الحالات، ومن الواضح أن هذه لـأن الارتباطات

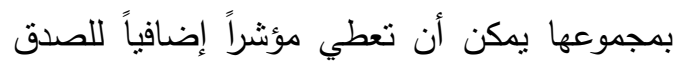

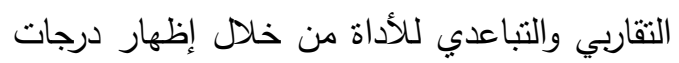

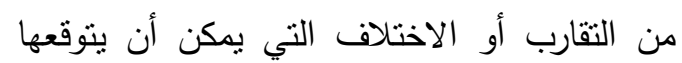

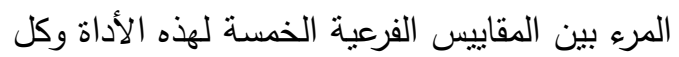
من سمتي الانبساط والعصابية، هذا مع الإشارة إلى
ويظهر الجدول (ع) ارتباطات موجبة للمقاييس

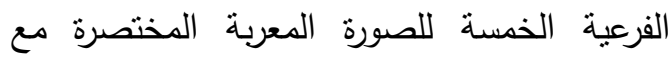
مقياس الانبساط (9 من أصل · ( ارتباطات منها

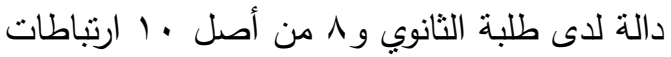
منها دالة لدى طلبة الجامعة)، وقد بلغ وسيط هذه

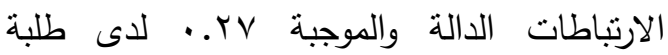
الثانوي وهب.. لاى طلبة الجامعة، في الوقت نفسه يظهر هذا الجدول ارتباطات سالبة لهذه

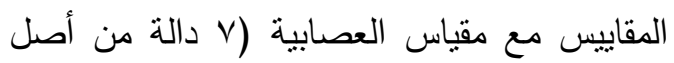

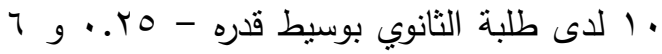
دالة من أصل · ا لاى طلبة الجامعة بوسيط قدره 
للإجابة عن السؤال الثالث من أسئلة الدراسة

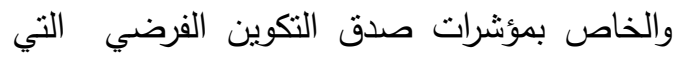
يمكن أن تعطيها الاداة درست الترابطات " البينية" للمقاييس الفرعية الخمسة التي تتضمنها، وقد أُجريت الدراسة على المجموعات السابقة نفسها التئي استخدمت في دراسة الصدق التقاربي والتباعدي للأداة، وهي مجموعات مختلطة (من الذكور والإناث) من طلبة الصف الثاني الثانوي والسنة الجامعية الثانية (قسم علم الاجنماع). وتظهر في الجدول (0) النتائج الخاصة بهذه الدراسة .
أن الارتباطات السالبة وغير الدالة مع مقياس

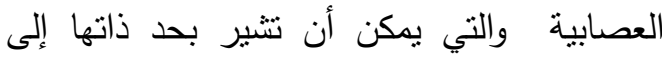
علاقة واهية لمجالات الرضا عن الحباة بالعصابية؛

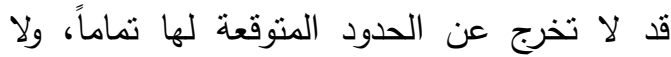

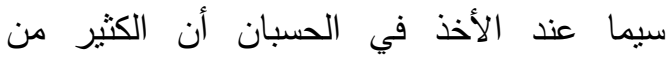
الأشخاص الأقل رضا عن الحياة قد لا بيتسمون بالعصابية بدرجة أكبر من الأثخاص الأكثر رضا. r- - النتائج الخاصة بالسؤال الثالث:

\section{الجدول (0)}

معملات الارتباط البينية للمقاييس الفرعية الخمسة للصورة المختصرة

\begin{tabular}{|c|c|c|c|c|c|c|c|c|c|c|c|c|}
\hline \multicolumn{6}{|c|}{ طلبة الجامعة } & \multicolumn{6}{|c|}{ طلبة الثانوية } & \multirow[b]{2}{*}{ ت الفرعية } \\
\hline 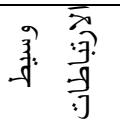 & $\begin{array}{l}=ن \\
V I\end{array}$ & ن & די & $\begin{array}{l}= \\
10\end{array}$ & $\begin{array}{l}=0 \\
V Y\end{array}$ & 录青争 & $\begin{array}{l}=0 \\
=\end{array}$ & ن & $\begin{array}{l}=0 \\
14\end{array}$ & $\begin{array}{l}=0 \\
V Y\end{array}$ & ن & \\
\hline דו & $\begin{array}{r}00 \\
1\end{array}$ & $\begin{array}{r}\cdot r \\
4\end{array}$ & מ מ. &.$r{ }^{2}$ & $\begin{array}{r}-r \\
q\end{array}$ &..$\leqslant 9$ & $\begin{array}{r}.0 \\
\varepsilon\end{array}$ & $\begin{array}{r}00 \\
1\end{array}$ & $\begin{array}{r}\cdot r \\
q\end{array}$ & $\begin{array}{r}\cdots \varepsilon \\
q\end{array}$ & $\begin{array}{r}\cdot \varepsilon \\
\varepsilon\end{array}$ & الأسرة مع الأصدقاء \\
\hline$\cdot . \leqslant Y$ & $\begin{array}{r}\cdot r \\
r\end{array}$ & $\begin{array}{r}\cdot \varepsilon \\
r\end{array}$ & .07 & .. $\leqslant 1$ & $\begin{array}{r}0.0 \\
1\end{array}$ &. $.7 r$ & $\begin{array}{c}\cdot r \\
r\end{array}$ & $\begin{array}{r}\cdot .7 \\
\varepsilon\end{array}$ & $\begin{array}{c}.7 \\
r\end{array}$ & $\begin{array}{r}\cdot \varepsilon \\
1\end{array}$ & $\begin{array}{c}0.0 \\
1\end{array}$ & الأسرة مع المدرسة \\
\hline. .09 & $\begin{array}{r}-0 \\
9\end{array}$ & $\begin{array}{r}\cdot r \\
q\end{array}$ & $\cdot . \leqslant r$ &. .09 & $\begin{array}{c}-7 \\
1\end{array}$ &. .01 & $\begin{array}{c}\ldots \\
1\end{array}$ & $\begin{array}{r}.0 \\
1\end{array}$ & $\begin{array}{c}\cdots \\
r\end{array}$ & $\begin{array}{r}.7 \\
.\end{array}$ & $\begin{array}{c}.0 \\
V\end{array}$ & الأسرة مع بيئة الحياة \\
\hline.$r \wedge$ & $\begin{array}{c}\cdot .1 \\
r\end{array}$ & $\begin{array}{r}\cdot r \\
V\end{array}$ & $\cdots \varepsilon-$ &.$r \Lambda$ & $\begin{array}{r}\cdot r \\
1\end{array}$ & 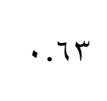 & $\begin{array}{c}\cdot .7 \\
r\end{array}$ & $\begin{array}{r}\cdot .7 \\
\varepsilon\end{array}$ & $\begin{array}{r}-7 \\
7\end{array}$ & $\begin{array}{r}\cdot . \varepsilon \\
q\end{array}$ & $\begin{array}{c}\cdot . \varepsilon \\
r\end{array}$ & الأسرة مع الذات \\
\hline .. $\varepsilon$. & $\begin{array}{c}. . \varepsilon \\
.\end{array}$ & $\begin{array}{r}.0 \\
1\end{array}$ &. .49 &. .00 & $\begin{array}{r}r \varepsilon \\
\varepsilon\end{array}$ &..$\leqslant 1$ & $\begin{array}{c}\ldots \varepsilon \\
1\end{array}$ & $\begin{array}{r}. . \varepsilon \\
.\end{array}$ & $\begin{array}{c}. . \varepsilon \\
r\end{array}$ & $\begin{array}{r}\cdot r \\
0\end{array}$ & $\begin{array}{c}.0 \\
r\end{array}$ & الأصدقاء مع المدرسة \\
\hline. .49 & $\begin{array}{c}. r \\
q\end{array}$ & $\begin{array}{r}\cdots \varepsilon \\
0\end{array}$ & .04 & זr. & r & (דז & $\begin{array}{r}. r \\
7\end{array}$ & $\begin{array}{r}\cdot \varepsilon \\
1\end{array}$ & $\begin{array}{r}. r \\
0\end{array}$ & & $\begin{array}{r}\cdots \varepsilon \\
\varepsilon\end{array}$ & الأصدقاء ع بيئة \\
\hline.. $\mathrm{H}$ & $\begin{array}{r}. r \\
V\end{array}$ & $\begin{array}{r}.0 \\
1\end{array}$ &. .01 &..$\Lambda$ &.$r$ &. .00 & $\begin{array}{r}.0 \\
0\end{array}$ & $\begin{array}{r}\cdot \cdot \\
\varepsilon\end{array}$ & $\begin{array}{r}.0 \\
1\end{array}$ & $\begin{array}{r}.0 \\
7\end{array}$ & $\begin{array}{r}\cdot r \\
\wedge\end{array}$ & الأصدقاء مع الذات \\
\hline.$\mu$ & $\begin{array}{l}\cdots \\
r\end{array}$ & $\begin{array}{r}\cdot r \\
r\end{array}$ &.$r \Lambda$ & $\cdot . \cdot V-$ & $\begin{array}{r}. r \\
r\end{array}$ &. .09 & $\begin{array}{r}.0 \\
9\end{array}$ & $\begin{array}{r}. r \\
9\end{array}$ & $\begin{aligned} \cdots \varepsilon \\
r\end{aligned}$ & $\begin{array}{r}1.0 \\
9\end{array}$ & $\begin{array}{c}\because .7 \\
.\end{array}$ & المدرسة مع بيئة الحياة \\
\hline$\cdot . \leqslant \varepsilon$ & $\begin{array}{r}. r \\
y\end{array}$ & $\begin{aligned} \cdot r \\
V\end{aligned}$ & $\cdot . \leqslant \varepsilon$ & $\cdot . \leqslant 9$ & $\begin{array}{r}.0 \\
r\end{array}$ &..$\leqslant 7$ & $\begin{array}{c}. . \varepsilon \\
.\end{array}$ & $\begin{array}{r}. . \varepsilon \\
7\end{array}$ & $\begin{array}{r}.0 \\
r\end{array}$ & $\begin{array}{r}\cdots \varepsilon \\
7\end{array}$ & $\begin{array}{c}-.0 \\
1\end{array}$ & المدرسة مع الذات \\
\hline..$\Lambda$ & 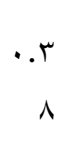 & $\begin{array}{r}\cdots \\
r\end{array}$ &.$r V$ & $\cdot 1 \cdot-$ & $\begin{array}{r}-1 \\
7\end{array}$ &. .49 & $\begin{array}{c}\bullet \varepsilon \\
.\end{array}$ & $\begin{array}{r}\cdot r \\
9\end{array}$ & $\begin{array}{r}\cdots \varepsilon \\
\varepsilon\end{array}$ & $\begin{array}{r}- \\
.1 \\
.\end{array}$ & $\begin{array}{r}\cdot r \\
v\end{array}$ & بيئة الحياة مع الذات \\
\hline
\end{tabular}


الارتباط المحسوبة بين كل مقياس وآخر لاى المجموعات الخمس من طلبة الجامعة تراوحت ما

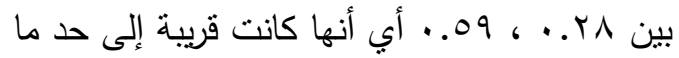
من الوسيطات المحسوبة لطلبة الثانوب، والتي وقعت في المدى من بr. من أمر فإنه يمكن القول استتاداً إلى النتائج السابقة بمجموعها : إن الترابطات البينية للمقاييس الفرعية الخمسة للصورة المختصرة لم تكن مرتفعة إلى الحد

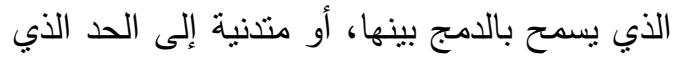

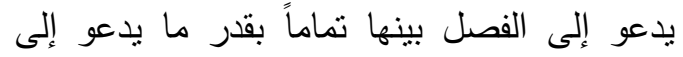

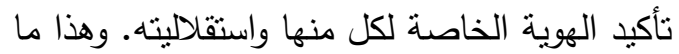
يتماشى مع النظرة التعددية التي ينطلق منها

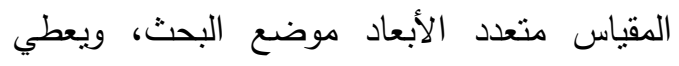
مؤشراً لصدق تكوينه الفرضي.

ومن الطرائق التي اتبعت أيضاً في دراسة صدق التكوين الفرضي للصورة المختصرة المعربة

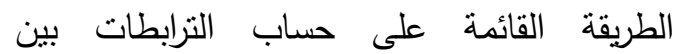
المقاييس الفرعية الخمسة لهذه الصورة والمقاييس الفرعية المناظرة لها للمقياس الكامل المعرب، وذللك استتاداً إلى أداء العينات الخمس المختلطة

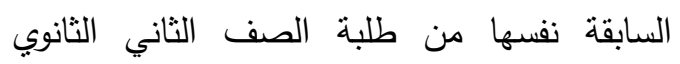
والسنة الجامعية الثانية (قسم علم الاجتماع). وتظهر نتائج هذه الدراسة في الجدول (7).
يتبين من الجدول (0) أن معاملات الارتباط البينية للمقاييس الفرعية الخمسة للصورة المختصرة

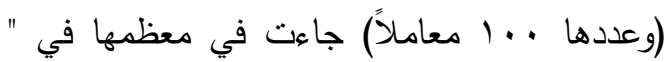

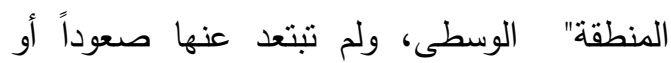
هبوطاً بمسافة كبيرة إلا في حالات قليلة، وبإمعان

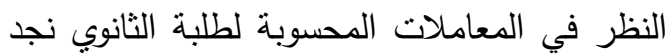
أنها لم تخرج عن الحدود المتوسطة، كما أنها دالة ( باستثناء معاملين اقتربا من الصفر أعطاهما ترابط الأسرة مع بيئة الحياة، وبلغت قيمة الأول منهما r +.. والثاني ( +..، ومعامل ثالث قريب من الصفر أعطاه ترابط الأصدقاء مع الذات وبلغت قيمته ؟ ...، ومعامل رابع سالب أعطاه نرابط بيئة الحياة مع الذات وبلغت قيمته - . ( . ). وقد تراوحت وسيطات معاملات الارتباط المحسوبة بين كل مقياس وآخر للى المجموعات الخمس السابقة تراوحت ما بين جr.. إلى باج..،

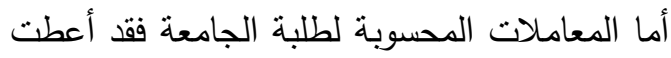
صورة مشابهة إلى حد كبير للصورة السابقة حيث

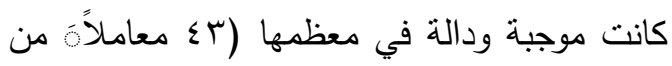

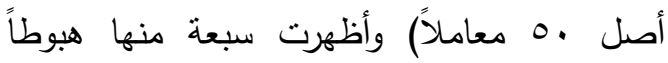
ملحوظاً أو كان سالباً (ثلاثة منها أعطاها نرابط

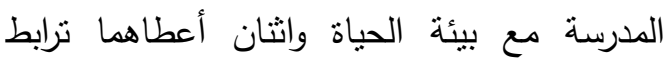
الأسرة مع الذات واثثان آخران أعطاهما ترابط بيئة الحياة مع الذات)، وقد تراوحت وسيطات معاملات

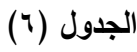

ترابطات مقاييس الصورة المختصرة مع مثيلاتها في المقياس الكامل

\begin{tabular}{|c|c|c|c|c|c|c|c|c|c|c|c|c|}
\hline \multicolumn{6}{|c|}{ طلبة الجامعة } & \multicolumn{6}{|c|}{ طلبة الثانوي } & \multirow{3}{*}{ المقاييس الفرعية } \\
\hline وسيط & ن & ن & ن & ن & ن & \multirow{2}{*}{ الارتباط } & \multirow{2}{*}{$\begin{array}{l}=0 \\
\text { TV }\end{array}$} & \multirow{2}{*}{ ن } & \multirow{2}{*}{ 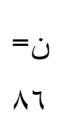 } & \multirow{2}{*}{$\begin{array}{l}= \\
V Y\end{array}$} & \multirow{2}{*}{ "זr } & \\
\hline الارتباطات & v) & Tr & 74 & 70 & VY & & & & & & & \\
\hline \multirow{2}{*}{$\because V V$} & \multirow{2}{*}{. .10} & \multirow{2}{*}{..$V V$} & \multirow{2}{*}{$\because V T$} & \multirow{2}{*}{. . } & \multirow{2}{*}{..$V \varepsilon$} & \multirow{2}{*}{.$V r$} &..$v$ &. .0 &. .7 &..$V$ &. .1 & الأسرة \\
\hline & & & & & & & $\varepsilon$ & q & 9 & r & 1 & \\
\hline \multirow{2}{*}{. .79} & \multirow{2}{*}{. .71} & \multirow{2}{*}{..$\vee 9$} & \multirow{2}{*}{. .74} & \multirow{2}{*}{..$V V$} & \multirow{2}{*}{. .79} & \multirow{2}{*}{..$v r$} & $\cdot . v$ &. .7 &.$v$ &..$v$ & $\therefore v$ & الأصدقاء \\
\hline & & & & & & & 1 & $\wedge$ & $r$ & $\wedge$ & $\varepsilon$ & \\
\hline
\end{tabular}




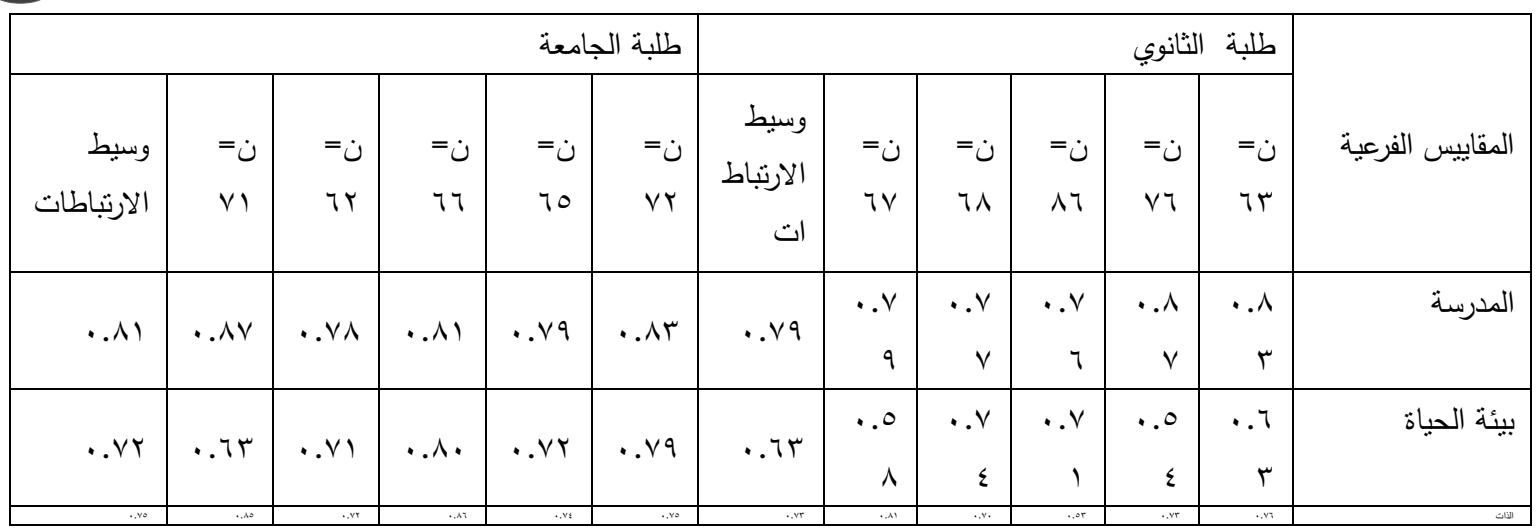

تربطه بيقية المقاييس، كما يظهر أن مقاييس

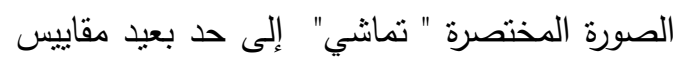

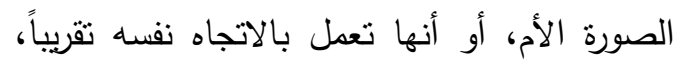

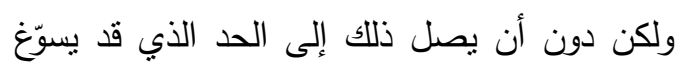
إحلال مقاييس الصورة المختصرة محل الطويلة، أو الاستغناء عن هذه الأخيرة لصالح الصورة المختصرة، وبطبيعة الحال فإن النتيجة السابقة تعطي مؤشراً مهما لصدق التكوين الفرضي للصورة

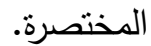

والطريقة الأخيرة التي انبعت في دراسة صدق

الصورة المختصرة تمت عن طريق حساب ترابط كل من المقاييس الفرعية التي تتضمنها مع درجات أفراد المجموعات السابقة من طلبة الصف الثاني الثانوي العام في امتحانات الفصل الأول من العام

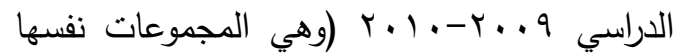
التي استخدمت في دراسة الصدق بدلالة محك التك

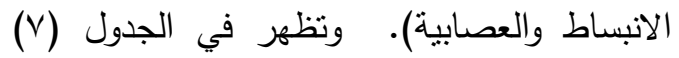
ترابطات المقاييس الفرعية الخمسة للصورة المختصرة مع الدرجات التحصيلية لأولئك الطلبة في تلاك الامتحانات.

(v) الجدول

ارتباطات المقاييس الفرعية الخمسة للصورة المختصرة مع الدرجات التحصيلية

\begin{tabular}{|c|c|c|c|c|c|c|}
\hline \multirow{2}{*}{ الارتباطات } & \multicolumn{5}{|c|}{ المجموعات } & \multirow[t]{2}{*}{ الدقاييس الفرعية } \\
\hline & ن & ن & ن & ن & ن= & \\
\hline **..Tr & **. . T。 & $* . . T V$ & $. .1 \leqslant$ & **..Tr & \% & الأسرة \\
\hline ***.rq & $\ldots \wedge$ & \% . . & $* *, . \Gamma$ &. .11 & $* * . r q$ & الأصدقاء \\
\hline
\end{tabular}

يلاحظ من الجدول (T) أن معظم الارتباطات التي أعطنها المقاييس الفرعية للصورة المختصرة تخطت بوضوح الحدود المتوسطة ومالت إلى الارتفاع بدرجة ما، وأن القليل منها فقط لم يتجاوز الحدود المتوسطة، مع ظهور بعض الفروق بين طلبة وان الثانوي والجامعة في هذه الارتباطات، وقد بلغت لهن وسيطات هذه الارتباطات لدى طلبة الثانوي لV .V. لمقياس الأسرة (مقابل VV. . . لدى طلبة الجامعة)،

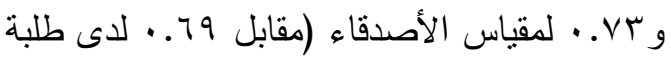

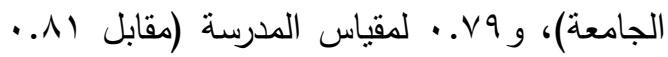
للى طلبة الجامعة)، و و 7.. ل لمقياس بيئة الحياة

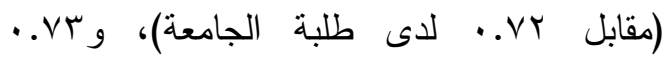
لمقياس الذات (مقابل ه v. . لدى طلبة الجامعة). وبمقارنة هذه الترابطات بالترابطات البينية لهذه المقاييس (أي الترابط بين كل مقياس فرعي وآخر ضمن الصورة المختصرة ذاتها) (الجدول ه ه يتبين أنها تخطتها بمسافة كبيرة وواضحة، وهذا ما يصب لهب في مصلحة الصورة المختصرة بطبيعة الحال، ويظهر قوة الصلة التي تربط كل مقياس فرعي بنظيره في الصورة الأم بالمقارنة مع الصلة التي لرني 


\begin{tabular}{|c|c|c|c|c|c|c|}
\hline \multirow{2}{*}{ الارتباطات } & \multicolumn{5}{|c|}{ المجموعات } & \multirow[t]{2}{*}{ المقاييس الفرعية } \\
\hline & ن & $\varepsilon q=\dot{ }$ & \{\urcorner$=\dot{ }$ & ن & ن= & \\
\hline •. & $* * . r V$ & $* *$. . & $* * \ldots \leqslant \Gamma$ & $* *$. . $\{9$ & **.r人 & المدرسة \\
\hline$* * . r \wedge$ & $* *$. . Y &. $.1 T$ & ד ש . & $* *$ *.r & *. . ro & بيئة الحياة \\
\hline$* . . r T$ & 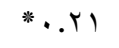 & $* . . r T$ & $* . Y Y$ & $* *$ *. Үᄉ & $*$ *.rl & الذات \\
\hline
\end{tabular}

الفرعية الخمسة لهذه الصورة، وترابطات هذه المقاييس الفرعية ذاتها مع مثيلاتها في المقياس الكامل (أو الصورة الأم المؤلفة من ـع بنداً)، ناهيك عن ارتباطها بالدرجات التحصبلية .

ولعل ما يضفي على النتائج السابقة شيئًا من الأهمية والجدة أنها تظهر كفاءة سيكومنرية مرضية للأداة عند استخدامها مع طلبة الجامعة، وهو مالم يكن موضع بحث في الدراسات السابقة للأداة، هذا بالإضافة إلى توفير المزيد من البيانات السيكومترية التي يمكن أن تتضم إلى البيانات السيكومترية المتجمعة حول الأداة استتاداً إلى أداء

طلبة المرحلة قبل الجامعية.

وبطبيعة الحال، فإن ما أظهرته هذه الأداة من توافق في دلالات الصدق التي أعطتها عند تطبيقها على مجموعات من طلبة الجامعة وطلبة الثانوي

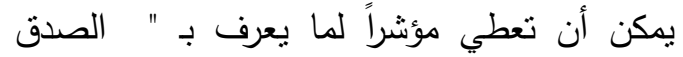

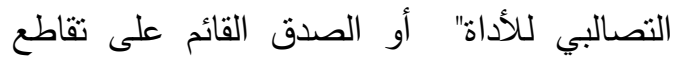
النتائج Cross-validatio ، وهو ما يوفر المزيد من الدعم لصدق الأداة موضع الاهتمام.

من خلال النتائج السابقة يرى الباحث

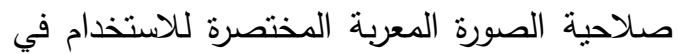

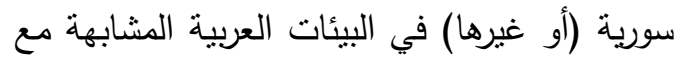
طلبة الثانوي والجامعة على حد سواء، وأن هذه الصورة يمكن أن تلبي الأغراض البحثية وأغراض المقارنات الجماعية والدراسات المسحية والحضارية المقارنة بكفاءة عالية بوصفها أداة سهلة الاستعمال و" اقتصادية" في الوقت والجهد، وبطبيعة الحال بهال
يتبين من الجدول السابق أن معاملات الارتباط

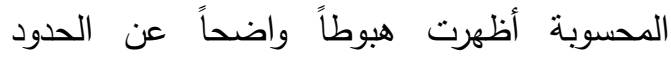
المتوسطة، ولكنها كانت دالة (باستثناء معامل واحد أعطاه مقياس الأسرة، وانثين أعطاهما مقياس الأصدقاء، ومعامل رابع أعطاه مقياس بيئة الحياة)، وقد نراوحت وسيطات معاملات الارتباط المحسوبة الإناه

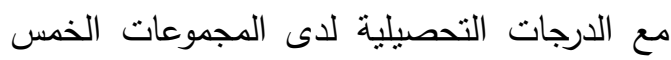

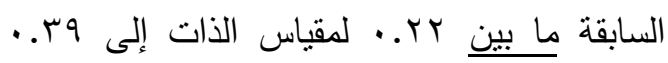
لمقياس المدرسة بوسيط قدره 9 ب..، ونتشير هذه الارتباطات إلى الصلة التي يمكن أن يتوقعها المرء

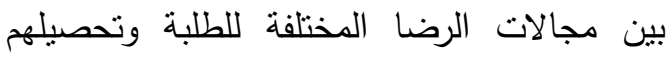
الدراسي، هذا مع الإثارة إلى أن مقياس الرضا عن الرنال

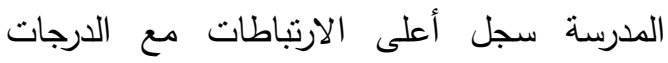
التحصيلية، وهو أمر متوقع، وقد يثبر إلى الدور

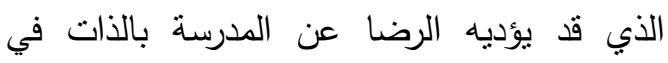

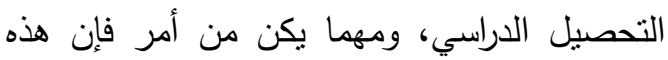
الارتباطات بمجموعها يمكن أن تؤخذ مؤشراً إضافياً لصدق الصورة العربية المختصرة. استتنتاجات ومقترحات : أظهرت نتائج الدراسة الحالية أن الصورة الصورة المعربة الموازية للصورة المختصرة لمقياس الرضا عن الحياة المتعدد الأبعاد للطالب BMSLSS أعطت مؤشرات سيكومترية بمكن وصفها بأنها مقبولة عموماً، وقد ظهر هذا عند دراسة ثنات

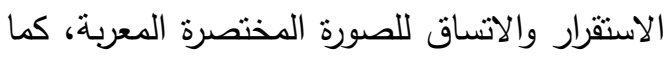
ظهر عند دراسة الصدق التقاربي والتباعدي لها بدلالة عدد من المقاييس المحكية المعتمدة، إضافة إلى دراسة الترابطات البينية (أو الداخلية) للمقاييس 
ويقترح الباحث إخضاع الصورة المعربة

المختصرة للمزيد من الدراسة والبحث، وذلك من

أجل الحصول على المزيد من البيانات السيكومترية والمعيارية التي يمكن أن ندعم ثناتها وصدقها، وسيكون من المفيد بطبيعة الحال استخدام أساليب

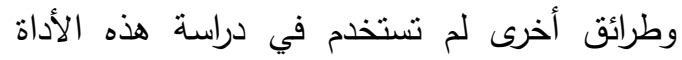
كطريقة الفرق المتقابلة (أو المجموعات المتضادة)

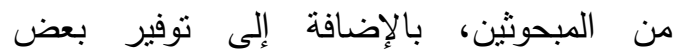
المؤشرات حول صدقها العاملي.
فإنه ليس هناك ما يمنع من حيث المبدأ من استخدام هذه الصورة في مجال التشخيص الفردي التفريقي ولكن شرط أن بقتصر ذلك على الحالات

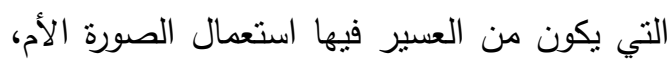
أو الحالات التي يسعى الفاحص إلى تكوين فكرة

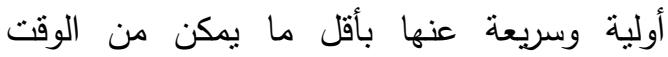

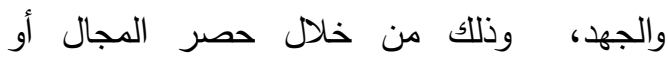
المجالات التي قد تبعث على الرضا (أو عدم الرضا) عند هذا الفرد أو ذالك .

\section{الكويتيين والعمانيين، مجلة العلوم التريوية والنفسية، 9(4) 107-131 .}

الدسوقي، مجدي محمد (999 (1). مقياس الرضا عن الحياة. القاهرة: مكتبة النهضة المصرية. عبد الخالق، أحمد محمد (1997) : دليل تعليمات القائمة العربية للتفاؤل والتشاؤم. الإسكندرية: دار المعرفة الجامعية.

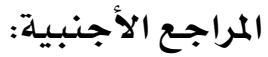

Adelman,H.S.,Taylor,L.,\&Nelson,P.(1989). Minors' dissatisfaction with their life circumstances. Child Psychiatry $\mathcal{E}$ Human Development,20. 135-147.

\&rews,F.M,\& Withey,S.B .(1976). Social indicators of well-being: Americans Perceptions of life quality. New York: Plenum.

Cantril,H.(1965). The patterns of human concern. New Brunswick, NJ: Rutgers University Press.

Diener, E. \& Diener, M. (1995). Cross cultural correlates of life satisfaction \&

\section{المراجع العربية: - المية}

عبد الخالق، أحمد محمد (997 (1)). دليل تعليمات قائمة بيك للاكتئاب. الإسكندرية: دار المعرفة

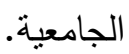

موسى، فاروق عبد الفتاح (1911). كراسة تعليمات اختبار الافعع للإنجاز للأطفال

والراشدين. القاهرة : مكتبة النهضة العربية

الأنصاري، بدر محمد، وكاظم، علي مهدي

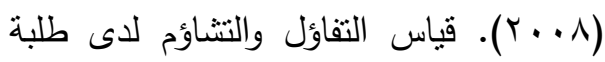
الجامعة: دراسة ثقافية مقارنة بين الطلبة

self-esteem. Journal of Personality $\mathcal{E}$ Social Psychology , 68,653-663.

Diener,E.,Emmons,R.A.,Larsen,R.J.,\& Griffin,S.(1985). The Satisfaction With Life Scale. Journal of Personality Assessment,49(1),71-75.

Digdem, M. S., \& Alim, K.(2008).Validity \& Reliability of the Brief Multidimensional Students' Life Satisfaction Scale With Turkish Children. Journal of

Psychoeducational Assessment,26(2),139147. Retrieved July,21,2008 from 
http://jpa.sagepub.com/cgi/ content/ abstract/ 26/2/139.

Gilman, R., Huebner, E. S., \& Laughlin, J.(2000). A first study of the Multidimensional Students' Life Satisfaction Scale with adolescents. Social Indicators Research, 52,135-160.

Greenspoon, P. J. \& Saklofske, D. H.(1997). Validity \& reliability of the Multidimensional Students' Life Satisfaction Scale with Canadian children. Journal of Psychoeducational Assessment, 15,138-155.

Huebner, E. S., Drane, J. W., \& Valois, R. F.(2000). Levels \& demographic correlates of adolescent life satisfaction reports. School Psychology International,21,281-292.

Huebner, E.S.(1991). Initial development of the Students' Life Satisfaction scale. School Psychology International, 12,231,243.

Huebner , E. S. ( 1994 ). Preliminary development \& validation of a multidimensional life satisfaction scale. Journal of Psychological Assessment , 6,149-158.

Huebner,E.S.(1995). The Students, Life Satisfaction scale: An assessment of psychometric properties with black \& white elementary school students. Social indicators research, 34,315-323.

Huebner,E.S.(2001). Manual for the Multidimensional Students, Life Satisfaction scale. University of South Carolina, Department of Psychology. Columbia, SC29208.

Huebner,E.S.,Laughlin , J.E.,Ash C.,\&Gilman , R.(1998).Further validation of the Multidimensional Students' Life Satisfaction Scale. Journal of Psychoeducational Assessment,16,118-134.

Huebner,E.S.,Suldo,S.,Valois,R.F.,Drane,J. W.\&Zullig.K.(2004).Brief

Multidimensional students life satisfaction scale: sex , race, \& grade effects for a high school sample. Psychological Reports. 94:351-356.
Huebner,E.S.,Suldo,S.M.,\&Valois,R.F.(2003 ).Psychometric Properties of Two Brief Measures of Children's Life satisfaction: The Students' Life satisfaction Scale(SLSS) \& the Brief Multidimensional Students' Life Satisfaction Scale(BMSLSS). Paper prepared for the Indicators of Positive Development Conference. March 1213,2003. Running Head :Life Satisfaction.

Kamman,R.,\&Flett,R.(1988).Affectometer2: A scale to measure current level of general happiness. Australian Journal of Psychology.35(2),259-265.

Lawton,M.P.(1975). The Philadelphia Geriatric Center Morale Scale: A revision. Journal of Gerontology, 30.8589.

Neugarten, B. L., Havighurst, R. J.,\& Tobin, S. S.(1961). The measurement of life satisfaction. Journal of Gerontology, $16,134-143$

Sam, D.L.(2001).Satisfaction with life among international students: An exploratory study. Social Indicators Ressearch.53,315-337.

Saunders, S. A \& Roy. C. (1999). The relationship between depression, Satisfaction with Life, \& social interest. South pacific Journal of Psychology, 11(1),9-15.

Seligson,J.L.,Huebner,E.S.,\&Valois,R.F.(20 03).Preliminary validation of the Brief Multidimensional Students' Life Satisfaction Scale(BMSLSS). Social Indicators Research,61,121-145.

Shin, D. C., \&Johnson, D. M. (1978). Avowed happiness as an overall assessment of the quality of life. Social Indicators Research,5,475-492.

Valois,R.F.,Zullig,K.J.,Huebner,E.S.,\&Dran e,J.W.(2001).Relationship between life satisfaction \& violent behaviors among adolescents. American Journal of Health Behavior,25(4),353-366.

Veenhoven,R.(1991).Is happiness relative?. Social Indicators Research,24, 1-34 


\section{(1) (الملحق )}

الصورة المختصرة لمقياس الرضا عن الحياة المتعدد الأبعاد للطلبة (BMSLSS) :

تدور العبارات التالية حول درجة الرضا (أو عدم الرضا) الذي قد يشعر به الطالب في مجالات مختلفة من

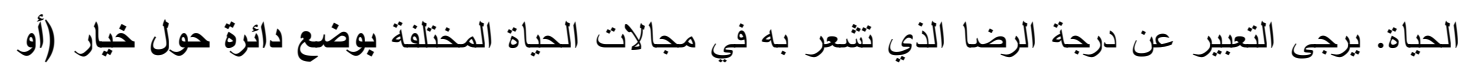

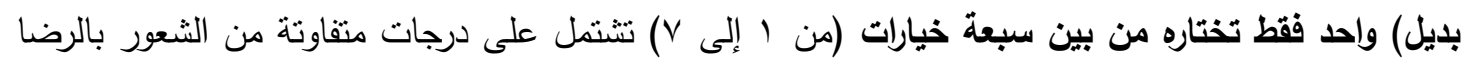

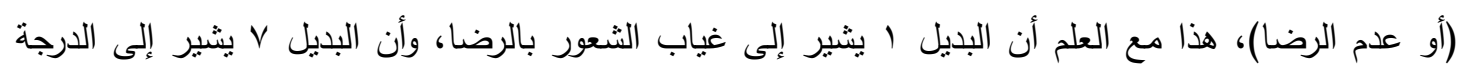

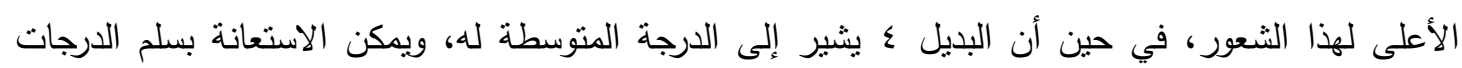
التالي في عملية الاختبار:

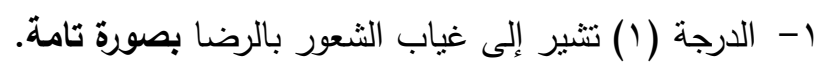

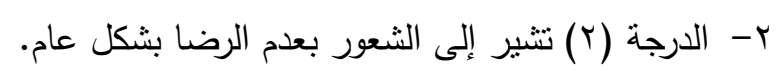
r- الدرجة (ץ) تشير إلى الثعور بعدم الرضا في أغلب الأحيان.

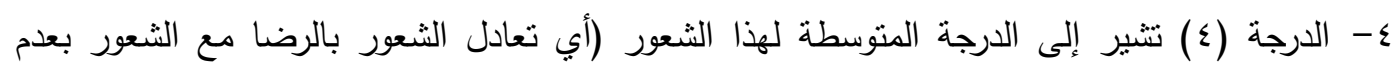

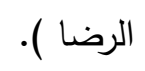

$$
\begin{aligned}
& \text { 0- الدرجة (0) تشير إلى الشعور بالرضا في أغلب الأحيان. } \\
& \text { ؟- الدرجة (ך) تشير إلى الثعور بالرضا بشكل عام. } \\
& \text { - الدرجة (V) تشير إلى الشعور بالرضا بصورة تامة. }
\end{aligned}
$$

\begin{tabular}{|c|c|c|c|c|c|c|c|}
\hline 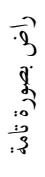 & $\begin{array}{l}\overrightarrow{3} \\
\frac{3}{3} \\
\frac{9}{2}\end{array}$ & 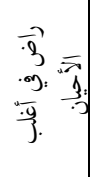 & 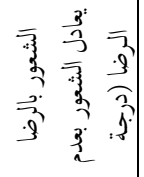 & 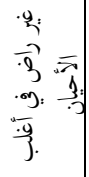 & $\begin{array}{l}\frac{9}{3} \\
\frac{3}{3} \\
3 \\
\frac{3}{3}\end{array}$ & $\begin{array}{l}\frac{9}{2} \\
\frac{3}{3} \\
\frac{3}{3}\end{array}$ & الأسئلة \\
\hline V & 7 & 0 & $\varepsilon$ & $r$ & $r$ & 1 & 1- أقدر أن شعوري بالرضا نحو أسرتي تعبر \\
\hline v & 7 & 0 & $\varepsilon$ & $r$ & $r$ & 1 & 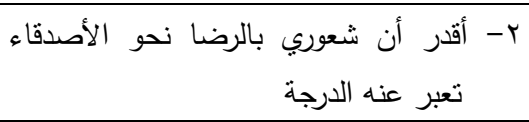 \\
\hline v & 7 & 0 & $\varepsilon$ & $r$ & $r$ & 1 & 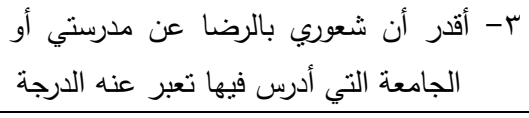 \\
\hline v & 7 & 0 & $\varepsilon$ & $r$ & $r$ & 1 & ع - أقدر أن شعوري بالرضا عن نفسي تعبر \\
\hline v & 7 & 0 & $\varepsilon$ & $r$ & $r$ & 1 & 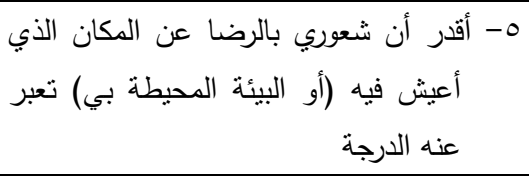 \\
\hline
\end{tabular}

(ملاحظة : لا يوجد إجابات صحيحة أو خاطئة هنا، ونرجو منك إعطاء الإجابة التي تعبر فعلاً عن حقيقة 